\title{
Component-graph construction
}

\author{
Nicolas Passat • Benoît Naegel · Camille Kurtz
}

the date of receipt and acceptance should be inserted later

\begin{abstract}
Component-trees are classical tree structures for grey-level image modelling. Componentgraphs are defined as a generalization of componenttrees to images taking their values in any (totally or partially) ordered sets. Similarly to component-trees, component-graphs are a lossless image model; then, they can allow for the development of various image processing approaches. However, component-graphs are not trees, but directed acyclic graphs. This makes their construction non-trivial, leading to non-linear time cost and resulting in non-linear space data structures. In this theoretical article, we discuss the notion(s) of component-graph, and we propose a strategy for their efficient building and representation, which are necessary conditions for further involving them in image processing approaches.
\end{abstract}

Keywords Component-graph · Algorithmics · Mathematical morphology · Multivalued images

\section{Introduction}

The component-graph is a hierarchical image model developed in the framework of mathematical morphology [1]. Most hierarchical models proposed in this context are trees, i.e. rooted, non-directed, connected, acyclic graphs. Well-known examples of such trees are, for

Nicolas Passat (corresponding author)

Université de Reims Champagne-Ardenne, CReSTIC, France

E-mail: nicolas.passat@univ-reims.fr

Benoît Naegel

Université de Strasbourg, CNRS, ICube, France

E-mail: b.naegel@unistra.fr

Camille Kurtz

Université Paris-Descartes, LIPADE, France

E-mail: camille.kurtz@parisdescartes.fr instance, the component-tree [2], the tree of shapes [3] or the binary partition tree [4]. By contrast, the component-graph is not a tree, but a directed acyclic graph (it shares this property with other models, such as component hypertrees [5] and directed component hierarchies [6]). This data structure is then more complex to build, store and manipulate. However, it is also more powerful, since it allows us to generalize the paradigm of component-tree not only to images taking their values in totally ordered (i.e. grey-level) sets, but in any (possibly partially) ordered sets. It then encompasses the spectrum of multivalued imaging.

After a preliminary study of the relations between component-trees and multivalued images [7], the notion of component-graph was introduced in [8]. A structural discussion was proposed in [9]. From an algorithmic point of view, first strategies for building componentgraphs were investigated. Except in a specific casewhere the partially ordered set of values is structured itself as a tree [10] — these first attempts emphasized a high computational cost of the construction process, and a high spatial cost of the directed acyclic graph explicitly modelling a component-graph $[11,12]$. By relaxing certain constraints, leading to an improved complexity, the notion of component-graph was involved in the development of an efficient extension of tree of shapes to multivalued images [13]. From an applicative point of view, the notion of component-graph, coupled with the recent notion of shaping [14], also led to preliminary, yet promising, results for multimodal image processing $[15,16]$.

The purpose of this theoretical study is to describe different variants of component-graphs, and to propose an algorithmic scheme for efficiently building the most relevant ones. By side effect, we also discuss the way to efficiently store the resulting component-graph. This 
article is an extended and improved version of the conference paper [17].

It is organised as follows. Sections 2 and 3 provide notations and basic notions. Sections 4 and 5 provide a discussion on various kinds of component-graphs. In particular, we motivate our choice of working in priority with a specific family called the strong componentgraphs. Sections 6-11 constitute the main contribution of the article. They discuss image preprocessing, data structure modelling and construction steps for the actual building and storage of a component-graph. Section 12 provides a complementary discussion about the space versus time cost trade-off to be handled for efficiently manipulating the component-graph.

\section{Notations}

We use the same notations as in [9-12].

The inclusion (resp. strict inclusion) relation on sets is noted $\subseteq$ (resp. $\subset$ ). The cardinality of a set $X$ is noted $|X|$. The power set of a set $X$ is noted $2^{X}$. If $\mathcal{P} \subseteq 2^{X}$ is a partition of $X$, we write $X=\bigsqcup \mathcal{P}$.

A function $F$ from a set $X$ to a set $Y$ is noted $F$ : $X \rightarrow Y$, and the set of all the functions from $X$ to $Y$ is noted $Y^{X}$. If $X^{\prime} \subseteq X$ and $Y^{\prime} \subseteq Y$, we note $F\left(X^{\prime}\right)=$ $\left\{F(x) \mid x \in X^{\prime}\right\}$ and $F^{-1}\left(Y^{\prime}\right)=\left\{x \in X \mid F(x) \in Y^{\prime}\right\}$. If $F$ is a bijection, we also note $F^{-1}: Y \rightarrow X$ its associated inverse function.

Let $\frown$ be a (binary) relation on a set $X$. The restriction of $\frown$ to a subset $Y \subseteq X$ will generally still be noted $\frown$.

We say that $\frown$ is an equivalence relation if $\frown$ is reflexive, transitive and symmetric. For any $x \in X$, the equivalence class of $x$ with respect to $\frown$ is noted $[x]_{\curvearrowright}$. The set of all these equivalence classes is noted $X / \frown$.

We say that $\frown$ is an order relation (and that $(X, \frown)$ is an ordered set) if $\frown$ is reflexive, transitive and antisymmetric. Moreover, we say that $\frown$ is a total (resp. partial) order relation (and that $(X, \frown)$ is a totally (resp. partially) ordered set), if $\frown$ is total (resp. partial) (i.e., if $\forall x, y \in X,(x \frown y) \vee(y \frown x)$ (resp. if $\exists x, y \in X,(x \nmid y) \wedge(y \nmid x)))$.

For any symbol further used to denote an order relation $(\subseteq, \leq, \unlhd$, etc.), the inverse symbol $(\supseteq, \geq, \unrhd$, etc.) denotes the associated dual order, while the symbol without lower bar $(\subset,<, \triangleleft$, etc.) denotes the associated strict order.

The Hasse diagram of an ordered set $(X, \leqslant)$ is the couple $(X, \prec)$ where $\prec$ is the cover relation associated to $\leqslant$, defined for all $x, y \in X$ by $x \prec y$ iff $x<y$ and there is no $z \in X$ such that $x<z<y$.

If $(X, \leqslant)$ is an ordered set and $x \in X$, we note $x^{\uparrow}=\{y \in X \mid y \geqslant x\}$ and $x^{\downarrow}=\{y \in X \mid y \leqslant x\}$,

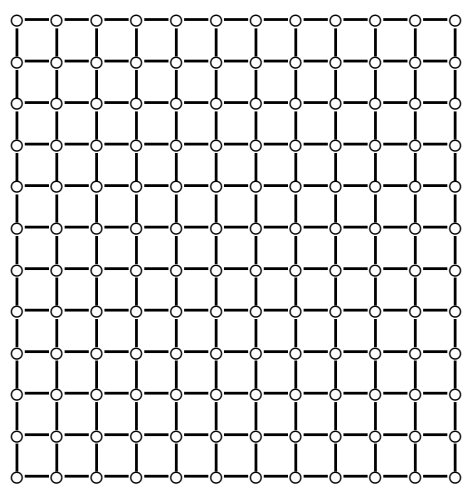

Fig. 1 A graph $(\Omega, \frown)$ where $\Omega$ is composed of 144 elements depicted by disks, whereas the adjacency relation $\frown$ between these elements is depicted by straight segments. This graph has the same structure as a $12 \times 12$ part of $\mathbb{Z}^{2}$ endowed with the standard 4-adjacency relation.

namely the sets of the elements greater and lower than $x$, respectively. If $Y \subseteq X$, the sets of all the maximal and minimal elements of $Y$ are noted $\nabla^{\leqslant} Y$ and $\Delta^{\leqslant} Y$, respectively. The supremum and the infimum of $Y$ are noted (when they exist) $\mathrm{V}^{\leqslant} Y$ and $\Lambda^{\leqslant} Y$, respectively (we will note $\bigcup$ and $\bigcap$ for $\bigvee^{\subseteq}$ and $\bigwedge^{\subseteq}$, respectively). The maximum and the minimum of $Y$ are noted (when they exist) $\gamma^{\leqslant} Y$ and $\Lambda^{\leqslant} Y$, respectively. If $Y$ is defined as $\{x \mid p(x)\}$ where $p$ is a Boolean predicate, we will sometimes note $\Upsilon_{p(x)}^{\leqslant} x$, instead of $\Upsilon^{\leqslant} Y$; the same remark holds for $\Lambda^{\leqslant}, V^{\leqslant}, \Lambda \leqslant, U, \cap, \sqcup$.

The symbol $\leq$ will be used to denote two distinct orders: the canonical order on $\mathbb{Z}$ and the pointwise order on functions; the context of use allows the reader to unambiguously associate the correct semantics to each occurrence of the symbol.

\section{Basic notions}

\subsection{Graph}

Let $\Omega$ be a nonempty finite set. Let $\frown$ be an adjacency (i.e. irreflexive, symmetric) relation on $\Omega$. The set $(\Omega, \frown)$ is then a non-directed graph. Let $X \subseteq \Omega$ be a subset of $\Omega$. The reflexive-transitive closure of (the restriction of) $\frown$ on $X$ induces the connectedness relation on $X$. It is an equivalence relation, and the set of the equivalence classes of $X$, called connected components, is noted $\mathcal{C}[X]$. Without loss of generality, we assume that $(\Omega, \frown)$ is connected, i.e. $\mathcal{C}[\Omega]=\{\Omega\}$.

Example 1 Figure 1 illustrates a graph $(\Omega, \frown)$ that represents a $12 \times 12$ part of $\mathbb{Z}^{2}$ endowed with the standard 4-adjacency relation. 


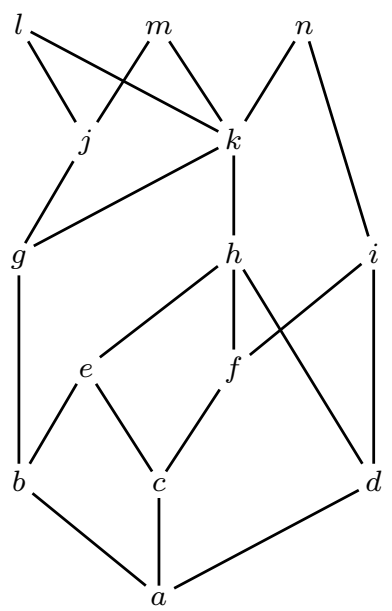

Fig. 2 Hasse diagram of a set $V=\{a, b, c, d, e, f, g, h, i, j, k$, $l, m, n\}$, equipped with a partial order relation $\leqslant$. The straight segments correspond to the cover relation $\prec$ associated to $\leqslant$. The greater a value, the higher in the graph. For instance, we have $h \prec k$, since $k$ is at a higher position than $h$. In particular, as $a=\perp$ is the minimum of $(V, \leqslant)$, it is at the lowest position. This ordered set does not have any maximum, but three maximal values $l, m$ and $n$.

\subsection{Image}

Let $\Omega$ be a non-directed graph, such as defined above. Let $V$ be a nonempty finite set equipped with an order relation $\leqslant$. We assume that $(V, \leqslant)$ admits a minimum, noted $\perp$. An image is a function $I: \Omega \rightarrow V$. The sets $\Omega$ and $V$ are called the support and the value space of $I$, respectively. For any $x \in \Omega, I(x) \in V$ is the value of $I$ at $x$. Without loss of generality, we assume that $I^{-1}(\{\perp\}) \neq \emptyset$. If $(V, \leqslant)$ is a totally (resp. partially) ordered set, we say that $I$ is a grey-level (resp. a multivalued) image.

Example 2 Figure 2 describes a partially ordered set $(V, \leqslant)$ composed of 14 values. More precisely, it illustrates the Hasse diagram $(V, \prec)$ associated to $(V, \leqslant)$. Figure 3 illustrates an image I defined on the support $\Omega$ of Figure 1, and taking its values in the ordered set $(V, \leqslant)$ of Figure 2 .

\subsection{Level-set image (de)composition}

Let $X \subseteq \Omega$ and $v \in V$. The thresholding function $\lambda_{v}$ is defined by

$$
\mid \begin{aligned}
\lambda_{v}: V^{\Omega} & \rightarrow 2^{\Omega} \\
I & \mapsto\{x \in \Omega \mid I(x) \geqslant v\}
\end{aligned}
$$

Example 3 A thresholded image is illustrated in Figure 4 .

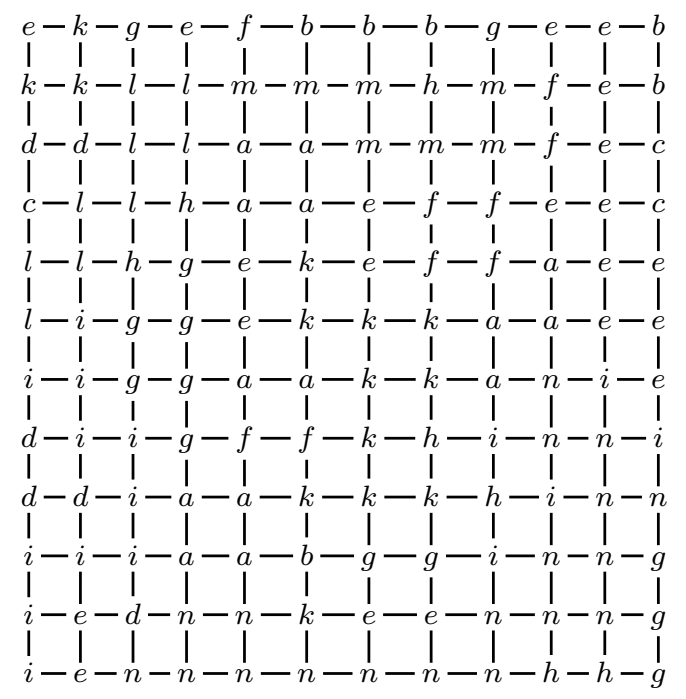

Fig. 3 An image $I: \Omega \rightarrow V$, where $(\Omega, \frown)$ is the graph of Figure 1 and $(V, \leqslant)$ is the ordered set of Figure 2. For the sake of readability, each element $x$ of $\Omega$ is replaced by $I(x)$. This image will be used for all the further illustrations of the article.

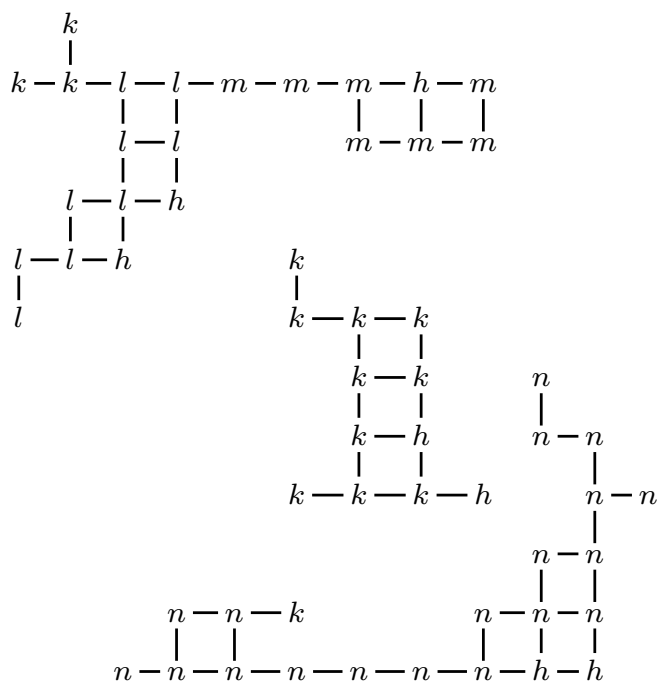

Fig. 4 The image $I$ of Figure 3 , thresholded at value $h$. More precisely, we show here the subgraph $\left(\lambda_{h}(I), \frown\right)$ (Equation (1)). The values $I(x)$ at the remaining points $x \in \lambda_{h}(I)$ are provided, for easing the comparison with Figure 3 . We can observe that $\left(\lambda_{h}(I), \frown\right)$ has three connected components.

The cylinder function $C_{(X, v)}$ is defined by

$$
\begin{aligned}
C_{(X, v)}: \Omega & \rightarrow V \\
x & \mapsto \begin{cases}v & \text { if } x \in X \\
\perp & \text { otherwise }\end{cases}
\end{aligned}
$$

In other words, a cylinder function is a bivalued piecewise constant function that associates the minimum value $\perp$ everywhere except on a given subset $X$ of the support where a chosen value $v$ is applied. 
Example 4 Trivial examples of cylinder functions are the functions $C_{(\{x\}, I(x))}$ for $x \in \Omega$. Such functions have the same value as $I$ at point $x$ and map each other points to $\perp$. The combination of these cylinder functions for all the $x \in \Omega$ allows us to construct $I$ as

$I=\bigvee_{x \in \Omega}^{\leq} C_{(\{x\}, I(x))}$

Remark 1 More generally, an image $I: \Omega \rightarrow V$ can be decomposed into cylinder functions induced by thresholding operations and, symmetrically, I can be reconstructed by composition of these cylinder functions

$I=\bigvee_{v \in V}^{\leq} \bigvee_{X \in \mathcal{C}\left[\lambda_{v}(I)\right]}^{\leq} C_{(X, v)}$

\section{Valued connected components and orders}

\subsection{Valued connected components}

We note $\Psi$ the set of all the connected components obtained from all the thresholdings of $I$

$\Psi=\bigcup_{v \in V} \mathcal{C}\left[\lambda_{v}(I)\right]$

Example 5 The whole support $\Omega$ is an element of $\Psi$, since it is a connected component (actually the unique one) of $\lambda_{\perp}(I)$, i.e. $\mathcal{C}\left[\lambda_{\perp}(I)\right]=\{\Omega\}$. The set $\mathcal{C}\left[\lambda_{h}(I)\right]$ is composed of three connected components (Figure 4 ). These three subsets of $\Omega$ are then also some elements of $\Psi$.

Remark 2 It may happen that a same set $X \subseteq \Omega$ belongs to distinct $\mathcal{C}\left[\lambda_{v}(I)\right]$ for various values $v \in V$. However, $X$ is only present once in $\Psi$, which is defined as a set, and not a multiset.

Remark 3 The set $\Psi$ of connected components does not preserve the information of the value $(s) v$ such that $X \in \mathcal{C}\left[\lambda_{v}(I)\right]$.

By assigning to $X \in \Psi$ a value $v \in V$ such that $X$ is a connected component of the level set $\lambda_{v}(I)$, we can define a notion of valued connected component.

Definition 1 (Valued connected component) Let $v \in V$ and $X \in \mathcal{C}\left[\lambda_{v}(I)\right]$. The couple $K=(X, v)$ is called a valued connected component; $X$ is the support of $K$ and $v$ is its value. We define the set $\Theta$ of all the valued connected components of $I$ as

$\Theta=\bigcup_{v \in V} \mathcal{C}\left[\lambda_{v}(I)\right] \times\{v\}$
Example 6 The valued connected component associated to the connected component $\Omega \in \Psi$ is $(\Omega, \perp) \in \Theta$. If $X \in \Psi$ is a connected component such that $X \in$ $\mathcal{C}\left[\lambda_{v}(I)\right]$ and $X \in \mathcal{C}\left[\lambda_{w}(I)\right]$ for $v \neq w$, then $X$ appears once in $\Psi$, whereas two corresponding valued connected components $(X, v)$ and $(X, w)$ appear in $\Theta$.

\subsection{Orders on valued connected components}

In the case of grey-level images, the connected components of $\Psi$ are equipped with the inclusion relation $\subseteq$. The component-tree is then defined as the Hasse diagram of the partially ordered set $(\Psi, \subseteq)$.

The purpose of component-graphs is to generalize this model to the case of images taking their values in sets which are not canonically equipped with total orders; this is for instance the case of multivalued images. This requires to define a relevant order relation $\unlhd$ on the set $\Theta$ of valued connected components, and to ensure that this relation remains compliant with $\subseteq$ in the case where $\leqslant$ is a total order on $V$. Under such conditions, the component-graph, defined as the Hasse diagram of $(\Theta, \unlhd)$, will be a relevant generalization of the component-tree.

Practically, there exist several ways to define a relation $\unlhd$ on $\Theta$ that takes into account the support of the valued connected components and / or their value, i.e. that involves $\subseteq$ and / or $\leqslant$.

In particular, five variants of $\unlhd$ can be proposed, in first intention, by composing $\subseteq$ and $\leqslant$ via standard policies (namely conjunction, lexicography, projection)

$$
\begin{aligned}
& (X, v) \unlhd_{1}(Y, w) \Leftrightarrow X \subseteq Y \\
& (X, v) \unlhd_{2}(Y, w) \Leftrightarrow(X \subset Y) \vee(X=Y \wedge w \leqslant v) \\
& (X, v) \unlhd_{3}(Y, w) \Leftrightarrow(X \subseteq Y) \wedge(w \leqslant v) \\
& (X, v) \unlhd_{4}(Y, w) \Leftrightarrow(w<v) \vee(w=v \wedge X \subseteq Y) \\
& (X, v) \unlhd_{5}(Y, w) \Leftrightarrow w \leqslant v
\end{aligned}
$$

In particular, for any $K, K^{\prime} \in \Theta$, we have

$$
\begin{aligned}
& K \unlhd_{3} K^{\prime} \Rightarrow K \unlhd_{2} K^{\prime} \Rightarrow K \unlhd_{1} K^{\prime} \\
& K \unlhd_{3} K^{\prime} \Rightarrow K \unlhd_{4} K^{\prime} \Rightarrow K \unlhd_{5} K^{\prime}
\end{aligned}
$$

However, on the one hand, neither $\unlhd_{1}$ nor $\unlhd_{5}$ are order relations. Indeed, they are reflexive and transitive, by not antisymmetric, in general. For instance, for distinct $X, Y$ and $v, w$, we have $(X, v) \unlhd_{1}(X, w)$ and $(X, w) \unlhd_{1}(X, v)$ whereas $(X, v) \neq(X, w)$; and $(X, v) \unlhd_{5}(Y, v)$ and $(Y, v) \unlhd_{5}(X, v)$ whereas $(X, v) \neq$ $(Y, v)$. On the other hand, $\unlhd_{4}$ is an order relation, but it does not relevantly take into account the support information carried by the elements of $\Theta$. Indeed, two valued connected components are mainly compared with 
respect to their respective values. Their supports are only considered for a common value, and the condition $X \subseteq Y$, in Equation (10), simply rewrites as $X=Y$, thus ensuring antisymmetry.

Finally, only two relevant order relations remain, namely $\unlhd_{2}$ and $\unlhd_{3}$. In the sequel, they will be called weak and strong orders on $\Theta$, respectively.

Definition 2 (Strong order, weak order) The strong order $\unlhd_{s}$ and the weak order $\unlhd_{w}$ on $\Theta$ are defined as follows

$$
\begin{aligned}
(X, v) \unlhd_{s}(Y, w) & \Leftrightarrow(X \subseteq Y) \wedge(w \leqslant v) \\
(X, v) \unlhd_{w}(Y, w) & \Leftrightarrow(X \subset Y) \vee(X=Y \wedge w \leqslant v)
\end{aligned}
$$

The weak order is indeed a lexicographic order, whereas the strong order is a conjunctive order.

From Equation (12), we have the following property.

Property 1 Let $K, K^{\prime} \in \Theta$. We have

$$
K \unlhd_{s} K^{\prime} \Rightarrow K \unlhd_{w} K^{\prime}
$$

In other words, the strong order relation implies the weak one. The reverse is not true, in general. Indeed, when $X \subset Y$ (Equation (14)), the definition of strong ordering implies that $w \leqslant v$, while the weak ordering relaxes this constraint in that case (Equation (15)).

\section{Component-graphs}

In the sequel, the notation $\unlhd$ (and its derived notations) is used for dealing with both $\unlhd_{s}$ and $\unlhd_{w}$ (and their derived notations).

\subsection{General definitions}

The component-graph $\mathfrak{G}$ of an image $I: \Omega \rightarrow V$ is defined as the Hasse diagram of the ordered set $(\Theta, \unlhd)$. However, three variants of component-graphs can be relevantly considered by defining two additional subsets $\dot{\Theta}, \ddot{\Theta} \subseteq \Theta$ of valued connected components

$$
\begin{aligned}
& \dot{\Theta}=\bigcup_{X \in \Psi}\{X\} \times \bigvee\left\{v \mid X \in \mathcal{C}\left[\lambda_{v}(I)\right]\right\} \\
& \ddot{\Theta}=\bigcap\left\{\Theta^{\prime} \subseteq \Theta \mid I=\bigvee_{K \in \Theta^{\prime}}^{\leq} C_{K}\right\}
\end{aligned}
$$

Broadly speaking, $\Theta$ gathers all the valued connected components induced by $I ; \dot{\Theta}$ gathers the valued connected components of maximal values for any connected components; and $\ddot{\Theta}$ gathers the valued connected components associated to the cylinder functions which are sup-generators of $I$ (Equation (4)). A discussion and some illustrations of these three families of nodes can be found in [9].

Remark 4 The definition of $\Theta, \dot{\Theta}$ and $\ddot{\Theta}$ is independent from the choice between strong $\unlhd_{s}$ and weak order $\unlhd w$.

We note 4 (resp. $\dot{\triangleleft}$, resp. $\ddot{\triangleleft})$ the cover relation associated to the order relation $\unlhd$ on $\Theta$ (resp. to the restriction of $\unlhd$ to $\dot{\Theta}$, resp. to the restriction of $\unlhd$ to $\ddot{\Theta})$. From these definitions, we have

$\ddot{\Theta} \subseteq \dot{\Theta} \subseteq \Theta$

and

$\stackrel{\unlhd}{\bigvee} \Theta=\stackrel{\unlhd}{Y} \dot{\Theta}=\stackrel{\unlhd}{ } \ddot{\Theta}=(\Omega, \perp)$

$\stackrel{\unlhd}{\triangle} \Theta=\stackrel{\unlhd}{\triangle} \dot{\Theta}=\stackrel{\unlhd}{\triangle} \ddot{\Theta}$

In other words, despite decreasing cardinalities from $\Theta$ to $\ddot{\Theta}$, the (unique) maximum always remains the valued connected component $(\Omega, \perp)$ obtained when thresholding the whole support at the lowest value, whereas the minimal elements are always the same, due to the fact that the are necessarily the sup-generators of the image.

Remark 5 Equations (19-21) are valid for both strong $\unlhd_{s}$ and weak order $\unlhd_{w}$.

We have the following definition for the three variants of component-graphs.

Definition 3 (Component-graph(s)) Let $I: \Omega \rightarrow$ $V$ be an image. The $\Theta-($ resp. $\dot{\Theta}-$, resp. $\ddot{\Theta}-)$ componentgraph of $I$ is the Hasse diagram $\mathfrak{G}=(\Theta, \varangle)$ (resp. $\dot{\mathfrak{G}}=(\dot{\Theta}, \dot{\mathbf{4}})$, resp. $\ddot{\mathfrak{G}}=(\ddot{\Theta}, \ddot{\mathbf{\triangleleft}}))$ of the ordered $\operatorname{set}(\Theta, \unlhd)$

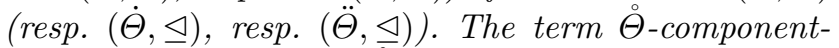

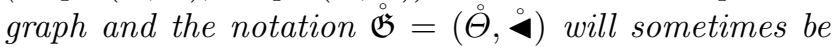
used to unify the three kinds of component-graphs. The

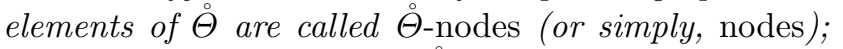

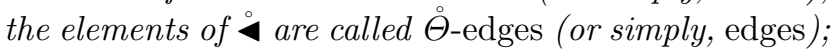
$(\Omega, \perp)$ is called the root; the elements of $\Delta^{\unlhd} \Theta$ are

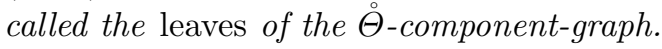

\subsection{Strong versus weak component-graphs}

When a component-graph is defined from the strong (resp. weak) order relation $\unlhd_{s}\left(\right.$ resp. $\left.\unlhd_{w}\right)$, it is called a strong (resp. weak) component-graph, and it is noted $\stackrel{\circ}{\mathfrak{G}}_{s}\left(\operatorname{resp} . \mathfrak{G}_{w}\right)$.

Example 7 Figures 5 and 6 illustrate the strong and weak component-graphs $\mathfrak{G}_{s}$ and $\mathfrak{G}_{w}$ of the image $I$ depicted in Figure 3. 


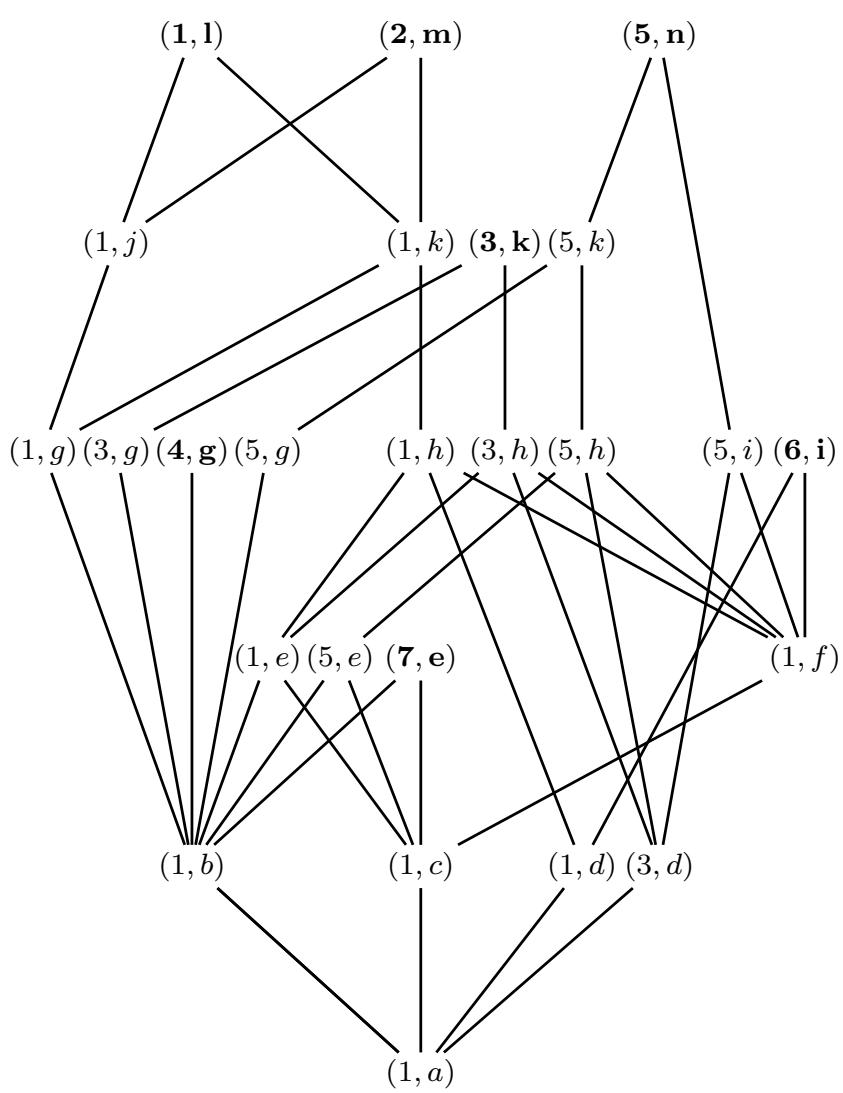

Fig. 5 The strong component-graph $\mathfrak{G}_{s}$ of the image $I$ of Figure 3. Each couple $(\ell, v)$ corresponds to a given node / valued connected component of value $v$, obtained as a connected component of the image $I$ thresholded at $v$; for instance, $(1, h),(3, h)$ and $(5, h)$ correspond to the three connected components of the subgraph $\left(\lambda_{h}(I), \frown\right)$ of Figure 4. The meaning of the labels $\ell$ will be explained in Section 10; at this stage, labels are only used to differentiate the nodes. The nodes in bold correspond to leaves of the component-graph. We can observe that for each node, the part of the componentgraph located below has exactly the same structure as the corresponding part of the Hasse diagram of $(V, \leqslant)$ (Equation (25)). Note that, contrary to the convention adopted in Figure 2, the higher the nodes, the lower with respect to the order $\triangleleft_{s}$. This reverse choice will allow us to better visualize the links between the component-graphs and the Hasse diagram of $(V, \leqslant)$. In particular, the maximum $(1, a)$ (that corresponds to $(\Omega, \perp))$ is located at the lowest position.

In the previous literature on this topic (and in particular, in [9]), the only considered component-graphs were the weak ones. In this section, we discuss about the relevance of strong versus weak component-graphs. This discussion will lead us to conclude that the first family is indeed to be preferred to the second, due to its more regular structural properties, whereas both families have globally the same image modelling abilities.

First, it was observed in Section 5.1 that strong and weak component-graphs rely on the same sets of nodes.

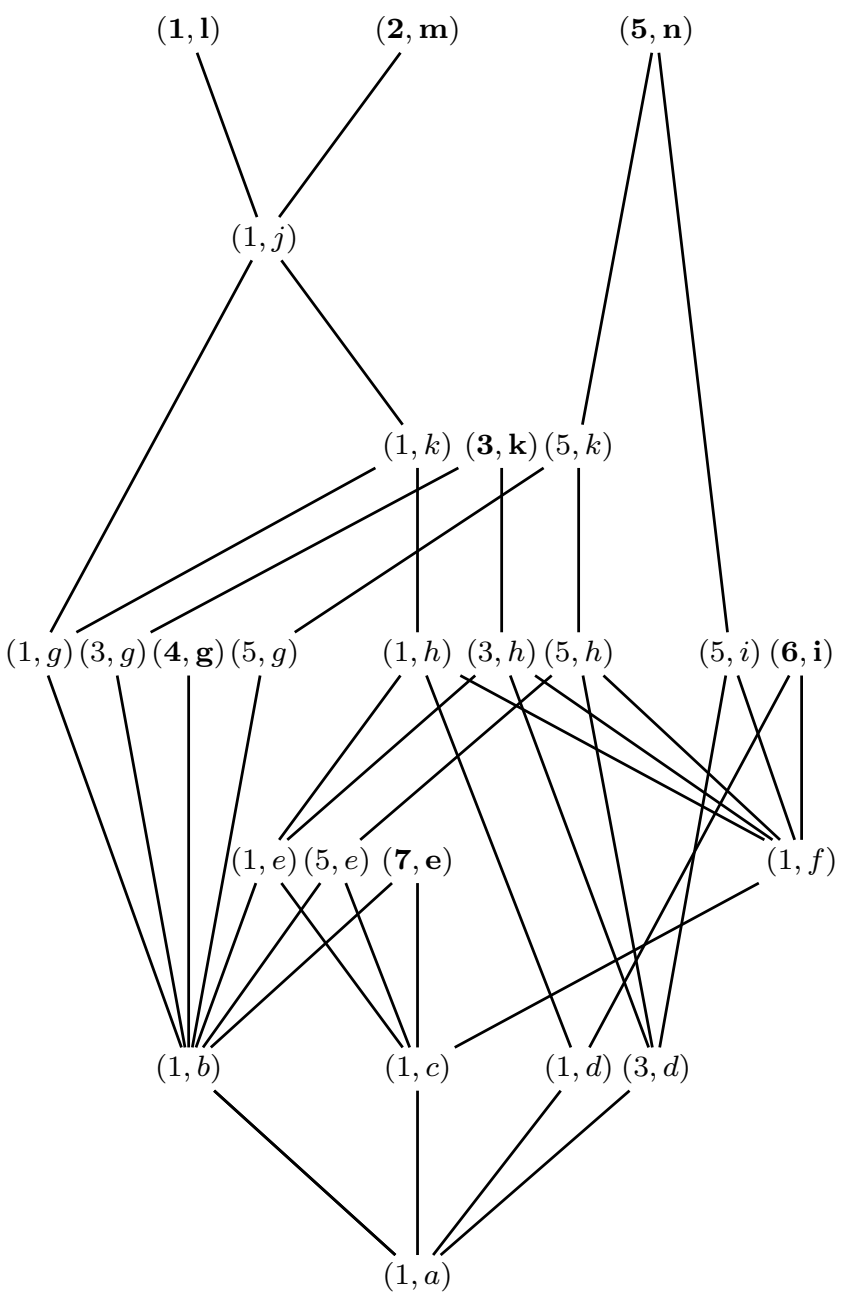

Fig. 6 The weak component-graph $\mathfrak{G}_{w}$ of the image $I$ of Figure 3 . By contrast with the strong component-graph, for each node, the part of the component-graph located below does not necessarily have the same structure as the corresponding part of the Hasse diagram of $(V, \leqslant)$ (Equation $(24)$ ). This is, for instance, the case below the nodes $(1, l)$ and $(2, m)$ where the nodes $(1, j)$ and $(1, k)$ are comparable with respect to $\unlhd_{w}$ due to the inclusion of their supports, whereas their values $j$ and $k$ are not comparable with respect to $\leqslant$

In particular, $\stackrel{\circ}{\mathfrak{G}}_{s}$ and $\mathfrak{\mathfrak { G }}_{w}$ have the same root, the same leaves, and the same subfamilies of nodes $\Theta, \dot{\Theta}$ and $\ddot{\Theta}$.

Second, both strong and weak component-graphs are relevant generalizations of the notion of componenttree. On the one hand, when $\leqslant$ is a total order, both notions are the same. On the other hand, the componentgraph is - such as the component-tree - a lossless image model with respect to the (de)composition formula of Equation (4). This is formalized in the following two propositions.

Proposition 1 Let $I: \Omega \rightarrow V$ be a grey-level image, i.e. $\leqslant$ is a total order on $V$. The component-tree $\mathfrak{T}$ of 
$I$ is isomorphic to its (strong and weak) componentgraphs $\dot{\mathfrak{G}}$ and $\ddot{\mathfrak{G}}$.

Remark 6 This isomorphism does not hold for $\mathfrak{G}$, in general, since $\mathfrak{T}$ gathers the same connected components obtained at successive values as a single node, contrary to $\mathfrak{G}$.

Proposition 2 Let $I: \Omega \rightarrow V$ be an image. We have

$I=\bigvee_{K \in \dot{\Theta}}^{\leq} C_{K}=\bigvee_{v \in V}^{\leq} \bigvee_{X \in \mathcal{C}\left[\lambda_{v}(I)\right]}^{\leq} C_{(X, v)}$

At this point, strong and weak component-graphs present the same properties. Their main difference, discussed hereinafter, actually lies in their respective structure with respect to the Hasse diagram $(V, \prec)$ of the partially ordered set $(V, \leqslant)$ of values.

As already observed in [9], the component-graph $\mathfrak{G}$ locally inherits from the structure of $(V, \prec)$. This is first stated by the following property.

Property $2([\mathbf{9}])$ Let $K:(X, v) \in \Delta^{\unlhd} \Theta$. The function

$\mid \begin{aligned} \sigma: K^{\uparrow} & \rightarrow v^{\downarrow} \\ (Y, w) & \mapsto w\end{aligned}$

is a bijection between $K^{\uparrow}$ and $v^{\downarrow}$.

Less formally, there is an exact mapping between the nodes of $\Theta$ located between the root $(\Omega, \perp)$ and a leaf $K=(X, v)$, and the values of $V$ located between the minimum $\perp$ and the value $v$.

However, this mapping does not take into account the way these nodes / values are organized. The difference appears when observing the relationships between $\leqslant, \unlhd_{s}$ and $\unlhd_{w}$ within the subgraphs associated to the leaves of the strong and weak component-graphs.

Property $3([\mathbf{9}])$ Let $K=(X, v) \in \Delta^{\unlhd} \Theta$. The function $\sigma^{-1}: v^{\downarrow} \rightarrow K^{\uparrow}$ induces a homomorphism from $\left(v^{\downarrow}, \geqslant\right)$ to $\left(K^{\uparrow}, \unlhd_{w}\right)$, i.e. for any $K_{1}=\sigma^{-1}\left(v_{1}\right), K_{2}=$ $\sigma^{-1}\left(v_{2}\right)$, we have

$\left(v_{1} \geqslant v_{2}\right) \Rightarrow\left(K_{1} \unlhd_{w} K_{2}\right)$

Property 4 Let $K=(X, v) \in \Delta^{\unlhd} \Theta$. The function $\sigma^{-1}: v^{\downarrow} \rightarrow K^{\uparrow}$ induces an isomorphism between $\left(v^{\downarrow}, \geqslant\right)$ and $\left(K^{\uparrow}, \unlhd_{s}\right)$, i.e. for any $K_{1}=\sigma^{-1}\left(v_{1}\right)$, $K_{2}=\sigma^{-1}\left(v_{2}\right)$, we have

$\left(v_{1} \geqslant v_{2}\right) \Leftrightarrow\left(K_{1} \unlhd_{s} K_{2}\right)$
In other words, the part of a component-graph located between the root $(\Omega, \perp)$ and each leaf $K=(X, v)$ contains some nodes that are directly associated to the values of $V$ located between $\perp$ and $v$. In the case of the strong component-graph, the structure of the Hasse diagram of $\left(K^{\downarrow}, \unlhd_{s}\right)$ is exactly the same as the structure of the Hasse diagram of $\left(v^{\uparrow}, \geqslant\right)$. By contrast, in the case of the weak component-graph, this structure is not always the same, and depends in priority on the $\subseteq$ relation between the supports of the nodes.

Example 8 In the strong component-graph of Figure 5 , the nodes between the leaf labeled as $(1, l)$ (resp. $(2, m))$ and the root $(1, a)$ have exactly the same structure as the values located between $l$ (resp. $m$ ) and $a$ in the Hasse diagram of $(V, \leqslant)$ (Figure 2). By contrast, this is no longer true in the weak component-graph of Figure 6, where we can observe that $(1, j)$ is lower than $(1, k)$ for $\unlhd_{w}$ despite the fact that $j$ and $k$ are not comparable for $\leqslant$. This is due to the fact that the support of $(1, k)$ is greater than that of $(1, j)$ for $\subseteq$.

Since both strong and weak component-graphs rely on the same nodes, and are defined from order relations that are relevant (they depend on both $\subseteq$ and $\leqslant$ ) and compliant with the component-tree in the case of grey-level images, it is preferable to focus on the strong component-graph, that presents a closer similarity with the Hasse diagram of $(V, \leqslant)$. This will allow us to take advantage of this known data structure during the construction process.

Before concluding this structural study, it is worth mentioning that the strong and weak componentgraphs are the same under specific hypotheses.

Property 5 We have $\ddot{\mathfrak{G}}_{s}=\ddot{\mathfrak{G}}_{w}$. In other words, for $K, K^{\prime} \in \ddot{\Theta}$ we have

$\left(K \ddot{\bullet}_{s} K^{\prime}\right) \Leftrightarrow\left(K \ddot{\bullet}_{w} K^{\prime}\right)$

Property 6 If $(V, \leqslant)$ is a lattice ${ }^{1}$, then we have $\dot{\mathfrak{G}}_{s}=$ $\dot{\mathfrak{G}}_{w}$. In other words, for $K, K^{\prime} \in \dot{\Theta}$, we have

$\left(K \dot{\boldsymbol{\triangleleft}}_{s} K^{\prime}\right) \Leftrightarrow\left(K \dot{\boldsymbol{\triangleleft}}_{w} K^{\prime}\right)$

In the next sections, we describe how to build the (strong) component-graph of an image.

\section{Flat zones and image reduction}

Let $I: \Omega \rightarrow V$ be an image defined on the graph $(\Omega, \frown)$, and taking its values in the ordered set $(V, \leqslant)$. Our

1 This means that for all $v, w \in V$, the two elements $\bigvee \leqslant\{v, w\}$ and $\Lambda \leqslant\{v, w\}$ exist and belong to $V$. 


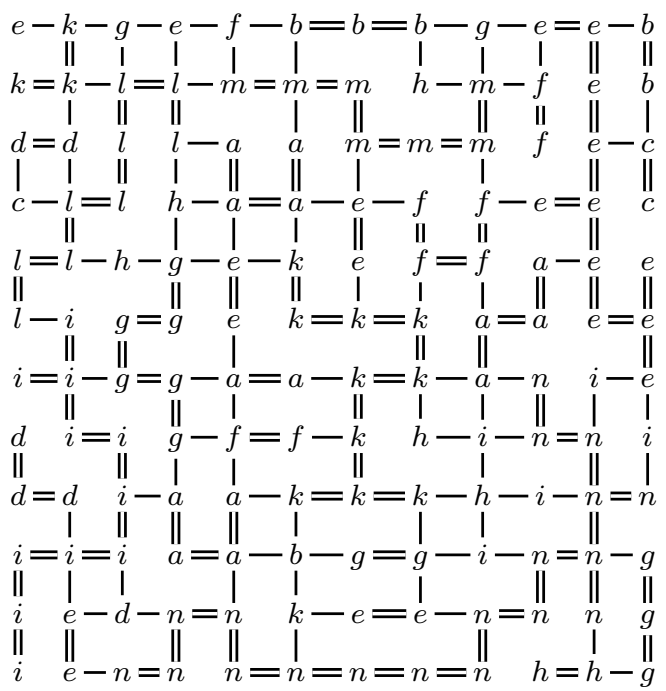

Fig. 7 The flat zone image $I_{\Phi}$ associated to the image $I$ of Figure 3. The maximal connected sets of same value linked by double edges - namely, the flat zones - correspond to the connected components for the equivalence relation $\leftrightarrow_{V}$. Each of these sets of $\Omega$ is then an element of $\Phi$. The adjacency relation $\frown_{\Phi}$ between two elements of $\Phi$ is depicted by a single edge between them. This adjacency $\frown_{\Phi}$ is inherited from the adjacency $\frown$ on $\Omega$ (Equation (30)).

purpose is to build the strong component-graph $\mathfrak{G}_{s}$ of I.

As a preliminary step, we show hereafter that a (weak or strong) component-graph is isomorphic to the component-graph of the flat zone image [18] associated to $I$. In the case of images where the number of flat zones is significantly lower than the number of points of the image support, this result will be useful for reducing the space and time cost of the algorithmic process of component-graph construction. Otherwise, this step is optional, and the remainder of the discussion (Section 7 and following) remains, of course, valid.

Let $v \in V$. Let $\frown_{v}$ be the relation defined on $\Omega$ as follows:

$(x \frown v y) \Leftrightarrow((x \frown y) \wedge(I(x)=I(y)))$

Let $\leftrightarrow_{v}$ be the reflexive-transitive closure $\frown_{v}$.

We define the relation $\leftrightarrow_{V}$ as the union of the $\leftrightarrow_{v}$, for all $v \in V$ :

$\left(x \leftrightarrow_{V} y\right) \Leftrightarrow\left(\exists v \in V, x \leftrightarrow_{v} y\right)$

The relation $\leftrightarrow_{V}$ is an equivalence relation that induces a partition of $\Omega$ with respect to the maximal connected sets of same value. These sets are called the flat zones.

Definition 4 (Flat zone) Let $I: \Omega \rightarrow V$ be an image. The set of the flat zones of $I$, noted $\Phi$ is the partition of $\Omega$ defined by $\Phi=\Omega / \leftrightarrow_{V}$.
Let us consider the relation $\frown_{\Phi}$ defined on $\Phi$, for any distinct $X, Y \in \Phi$, as follows:

$\left(X \frown_{\Phi} Y\right) \Leftrightarrow(\exists x \in X, \exists y \in Y, x \frown y)$

This relation $\frown_{\Phi}$ is the adjacency (irreflexive, symmetric) relation on the flat zones of $I$ induced by the adjacency relation on $\Omega$.

From the graph $\left(\Phi, \frown_{\Phi}\right)$, we can define the flat zone image $I_{\Phi}$ induced by $I$ as follows

$\begin{aligned} I_{\Phi}: \Phi & \rightarrow V \\ {[x]_{\leftrightarrow V} } & \mapsto I(x)\end{aligned}$

Example 9 Figure 7 illustrates the flat zone image $I_{\Phi}$ associated to the image I of Figure 3. In the upper-left part of this figure, one can observe three flat zones: a first composed of one point of value e, a second composed of three points of value $k$ and a third composed of two points of value $d$. These six points are grouped into three flat zones, locally leading to a reduction ratio of $\frac{1}{2}$ of the initial size of $\Omega$. The number of adjacency links is also reduced. For instance, there remains only one edge between the flat zone of value $k$ and the flat zone of value $e$ (resp. d) while there were two edges between the points of value $k$ and those of value e (resp. d) in the initial image.

We note $\Theta_{\Phi}=\bigcup_{v \in V} \mathcal{C}\left[\lambda_{v}\left(I_{\Phi}\right)\right] \times\{v\}$ the set of the valued connected components of $I_{\Phi}$. The following property is a direct consequence of the definitions of $\Phi$ and $\frown_{\Phi}$.

\section{Property 7 The function}

$\mid \begin{aligned} \phi: \Theta & \rightarrow \Theta_{\Phi} \\ (X, v) & \mapsto\left(X / \leftrightarrow_{V}, v\right)\end{aligned}$

is a bijection between $\Theta$ and $\Theta_{\Phi}$. Its inverse function is $\begin{aligned} \phi^{-1}: \Theta_{\Phi} & \rightarrow \Theta \\ (A, v) & \mapsto\left(\bigcup_{X \in A} X, v\right)\end{aligned}$

The following proposition establishes that we can work indistinctly on an image $I$ or its flat zone analogue $I_{\Phi}$, in order to build and use a component-graph.

Proposition 3 The bijection $\phi$ induces an isomorphism between the component-graphs $\stackrel{\circ}{\mathfrak{G}}=(\stackrel{\circ}{\Theta}, \stackrel{\mathbf{4}}{)})$ of $I$ and $\stackrel{\circ}{\mathfrak{G}}_{\Phi}=\left(\stackrel{\circ}{\Theta}_{\Phi}, \stackrel{\bullet}{\mathbf{4}}_{\Phi}\right)$ of $I_{\Phi}$. More precisely, we have

$((X, v) \stackrel{\circ}{\triangleleft}(Y, w)) \Leftrightarrow\left((\phi(X), v) \stackrel{\circ}{\triangleleft}_{\Phi}(\phi(Y), w)\right)$

Remark 7 The computation of $\left(\Phi, \frown_{\Phi}\right)$ from $(\Omega, \frown)$ can be done in linear time $\mathcal{O}(|\frown|)$. 


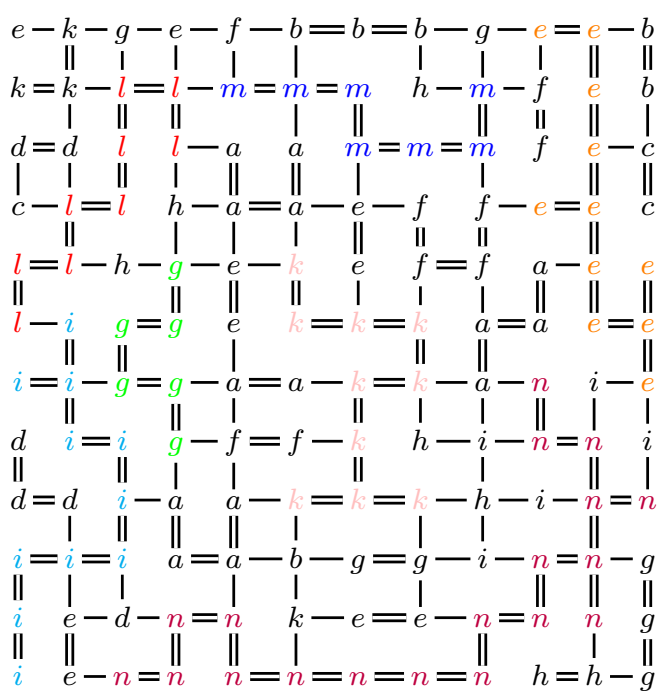

Fig. 8 The 7 leaves of the image $I$ of Figure 7 are depicted in colour. From now on, $I_{\Phi}$ will be noted as $I$ for the sake of concision. In particular, a flat zone is considered as a single element of $\Omega$ (formerly, $\Phi$ ). For instance, the blue flat zone composed of 7 points $x \in \Omega$ of value $I(x)=m$ is now a single point, and it is adjacent to 9 other elements of (lower or non-comparable) values $l, f, b, h, g, f, f, e$ and $a$. However, we keep the initial Cartesian grid formalism for the sake of readability and for allowing comparisons with Figures 3 and 7 .

Practically, it is then relevant to work with the flat zone image $I_{\Phi}$, where each flat zone of $I$, composed of $k \geq 1$ points, is replaced by an unique point. Doing so, the space complexity of the input image $I$ is reduced from $|\Omega|$ to $|\Phi|$, with $|\Phi| \leq|\Omega|$ (and $|\frown| \leq\left|\frown_{\Phi}\right|$ ), and in the most favourable cases $|\Phi| \ll|\Omega|$ (and $|\frown| \ll\left|\frown_{\Phi}\right|$ ). This reduction of space complexity will also have an impact on the time cost of the first steps of the componentgraph construction process. (The last steps, that already proceed at the scale of regions of the image, are not impacted by the initial use of flat zones.)

From Equations (22) and (31), it follows that the information carried by the component-graph of $I_{\Phi}$ is the same as that carried by $I$. Any image processing operation based on $\mathfrak{G}$ can then be performed the same way on $\mathfrak{G}_{\Phi}$.

Remark 8 From now on, we will no longer consider the image I, but its flat zone image $I_{\Phi}$. For the sake of readability, we will still note $I$ instead of $I_{\Phi}$, and $\Omega$ instead of $\Phi$. In particular, we will assume that for a given (flat zone) image $I$, we have for any $x, y \in \Omega$

$(x \frown y) \Rightarrow(I(x) \neq I(y))$

Remark 9 In digital imaging, we have $|\frown|=\mathcal{O}(|\Omega|)$. For instance, with 4-, 8-, 6- and 26-adjacencies on $\mathbb{Z}^{2}$ and $\mathbb{Z}^{3}$, we have $|\frown|=k .|\Omega|$, with $k=2,4,3$ and 13 , respectively [19]. This generally still holds for the induced flat zone images. For the sake of concision, we will assume from now on that we have $|\frown|=\mathcal{O}(|\Omega|)$.

\section{Building the leaves of a component-graph}

We are now ready to start building a (strong) component-graph. The cornerstone of this algorithmic process is the notion of a leaf.

A leaf $L$ is a minimal element of $\mathfrak{G}$, i.e. $L \in \Delta^{\unlhd} \stackrel{\circ}{\Theta}$ (Definition 3 and Equation (21)).

Since $\Omega$ is finite and $\leqslant$ is antisymmetric, there exist one or many points $x \in \Omega$ such that for all $y \frown x$, we have $I(x) \nless I(y)$, i.e. $I(x) \in V$ is a locally maximal value of the image $I$. Then, it is plain that $L_{x}=(\{x\}, I(x))$ is a node of $\stackrel{\circ}{\mathfrak{G}}$, i.e. $L_{x} \in \stackrel{\circ}{\Theta}$.

Example 10 Figure 8 illustrates the leaves of the image I of Figure \%. In particular, this image presents seven leaves. Let us consider the leaf of value $g$, depicted in green. The corresponding flat zone / point has seven neighbours of values $h, e, a, f, a, i$ and $h$, respectively. Indeed, two of these neighbours have a value lower than $g$, namely a (twice) whereas the other 5 have a non-comparable value, namely $h$ (twice), e, $f$ and $i$. Note that two (or more) leaves can be adjacent. This is the case of the green one with the leaf of value $i$, depicted in cyan. When this happens, the values of these leaves are, of course, non-comparable.

Remark 10 The characterization of a leaf relies on a local criterion: it is sufficient to observe the values of the adjacent points of a candidate point of $I$. Then, we can compute all the leaves of $\mathfrak{G}$ by an exhaustive scanning of $(\Omega, \frown)$, with a linear time cost $\mathcal{O}(|\Omega|)$.

In the sequel, we will denote by $\Lambda \subseteq \Omega$ the set of all the points of $\Omega$ that correspond to supports of leaves. These points will be called leaf-points. In other words, we have

$$
\stackrel{\unlhd}{\unlhd} \stackrel{\ominus}{\Theta}=\left\{L_{x}=(\{x\}, I(x)) \mid x \in \Lambda\right\}
$$

The notion of a leaf is fundamental for understanding the structure of a component-graph, and then for building it. In particular, in the next section, we show that the support $\Omega$ of the image $I$ can be decomposed into regions, each region being associated to a specific leaf / leaf-point. From these regions, and more precisely the adjacency relation between them, it will be possible to build the strong component-graph. 


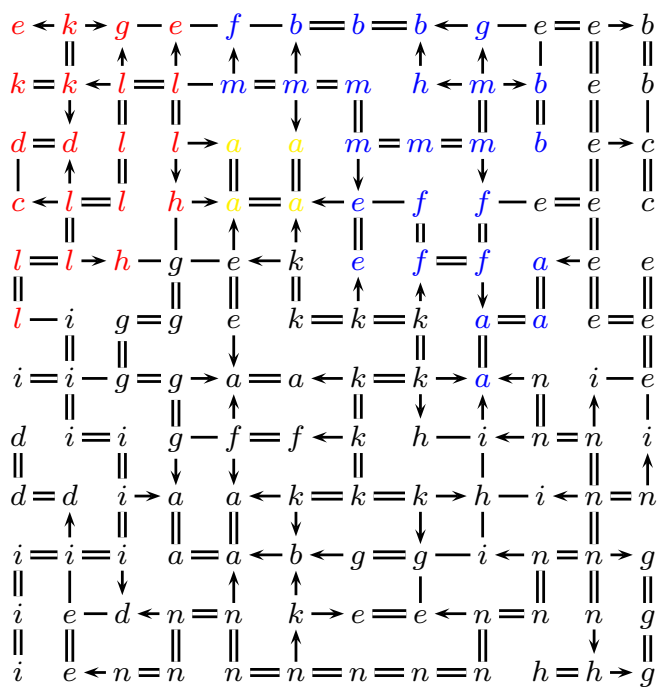

Fig. 9 Two reachable zones of the image $I$ of Figures 3 and 7. Here, an arrow pointing from an element $x \in \Omega$ of value $I(x) \in V$ to an element $y \in \Omega$ of value $I(y)$ indicates that $x$ and $y$ are adjacent (i.e. $x \frown y$ ) and $I(x)>I(y)$ (a segment with no arrow indicates that $x \frown y$ whereas $I(x)$ and $I(y)$ are non-comparable). A first reachable zone $\rho\left(x_{1}\right)$ is depicted in red and yellow for the leaf-point $x_{1} \in \Lambda$ of value $I\left(x_{1}\right)=l$. A second reachable zone $\rho\left(x_{2}\right)$ is depicted in blue and yellow for the leaf-point $x_{2} \in \Lambda$ of value $I\left(x_{2}\right)=m$. The overlapping part between $\rho\left(x_{1}\right)$ and $\rho\left(x_{2}\right)$ is depicted in yellow; here, it corresponds to only one point of value $a$.

\section{Influence zones of an image}

Each point $x \in \Omega$ has a given value $I(x) \in V$. It is also adjacent to other points $y \in \Omega$ (i.e. $x \frown y$ ) with values $I(y)$. From Equation (35), we have either $I(x)<I(y)$ or $I(x)>I(y)$ or $I(x), I(y)$ non-comparable.

From a leaf-point $x \in \Lambda$ corresponding to a leaf $L_{x}$, we can reach certain points $y \in \Omega$ by a descent paradigm. More precisely, for such points $y$, there exists a sequence $x=x_{0} \frown \ldots \frown x_{i} \frown \ldots \frown x_{t}=y(t \geq 0)$ in $\Omega$ such that for any $i \in \llbracket 0, t-1 \rrbracket$, we have $I\left(x_{i}\right)>$ $I\left(x_{i+1}\right)$. In such case, we note $x \searrow^{\Omega} y$. This leads to the following notion of a reachable zone.

Definition 5 (Reachable zone) Let $x \in \Lambda$ be a leafpoint of $I$. The reachable zone of $x($ in $\Omega$ ) is the set

$\rho(x)=\left\{y \in \Omega \mid x \searrow^{\Omega} y\right\}$

We note $P=\{\rho(x) \mid x \in \Lambda\}$ the set of all the reachable zones of $I$.

Example 11 Figure 9 illustrates the reachable zones of two leaf-points, namely the red one of value $l$ and the blue one of value $m$. Let us focus on the reachable zone of the first, red one. From this leaf-point of value l, one can directly reach reach eight other points of value $e, a$, $h, h, c, d, k$ and $g$, respectively. Of course, these eight values are strictly lower than $l$. From the point of value $k$, one can then reach another point of value $e$; this point then also belongs to the reachable zone. Note that a point can be reached from various distinct paths. This is the case of the one of value $d$ that is reached from a path of lenght 1 and another of length 2. In other words, the oriented graph of these reaching paths is a directed acyclic graph, but not a tree in general. Note also that a point can be reached from various leaves. This is the case of the yellow one, of value a, that can be reached from both the red and the blue leaf-points.

Property 8 The set $P$ is a cover of $\Omega$.

The important fact of this property is that $\bigcup_{x \in \Lambda} \rho(x)=$ $\Omega$. However, the set of reachable zones is not a partition of $\Omega$, in general, due to possible overlappings. For instance, let $x_{1}, x_{2} \in \Lambda, y \in \Omega$, and let us suppose that $x_{1} \frown y \frown x_{2}$ and $x_{1}>y<x_{2}$; then we have $y \in \rho\left(x_{1}\right) \cap \rho\left(x_{2}\right) \neq \emptyset$. This was already illustrated by the yellow point of Figure 9, discussed above.

Remark 11 The computation of $P$ can be carried out by a seeded region-growing process [20], with $\Lambda$ as set of seeds. The time cost is output-dependent, since it is linear with respect to the ratio of overlap between the different reachable zones. More precisely, it is $\mathcal{O}\left(\sum_{x \in \Lambda}|\rho(x)|\right)=\mathcal{O}((1+\gamma) .|\Omega|)$, with $\gamma \in[0,|\Omega| / 4] \subset$ $\mathbb{R}$ the overlap ratio, varying between 0 (no overlap between reachable zones, i.e. $\{\rho(x) \mid x \in \Lambda\}$ is a partition of $\Omega$ ) and $|\Omega| / 4$ (all reachable zones are maximally overlapped). The upper bound of $\gamma$ is $(|\Omega|-1)^{2} / 4|\Omega|$, and is reached when $|\Lambda|=(|\Omega|+1) / 2$.

Practically, in the worst cases, the time cost for computing $P$ is quadratic, namely $\mathcal{O}\left(|\Omega|^{2}\right)$. However, from an algorithmic point of view, we need not computing $P$ as a whole. Actually, we only need a partition $\Sigma$ of $\Omega$ that satisfies the following properties. This leads us to define a second notion of an influence zone.

Definition 6 (Influence zone) $A$ set of influence zones of $I$, noted $\Sigma$, is a partition of $\Omega$ defined such that for any $S \in \Sigma$, we have:

(i) $|\Lambda \cap S|=1$

(ii) $(x \in \Lambda \cap S) \Rightarrow(S \subseteq \rho(x))$

(iii) $\forall y \in S, x \searrow^{S} y$

In other words, there exists a unique leaf-point $x \in \Lambda \cap S$ and the set $S$ is included in the influence zone $\rho(x)$ of this unique leaf-point; in addition, any point $y$ of $S$ can be reached from $x$ by a descending path in $S$. For any leaf-point $x \in \Lambda$, the set $\sigma(x) \in \Sigma$ such that $x \in \sigma(x) \subseteq \rho(x)$ is called an influence zone of $x$ (in $I)$. 


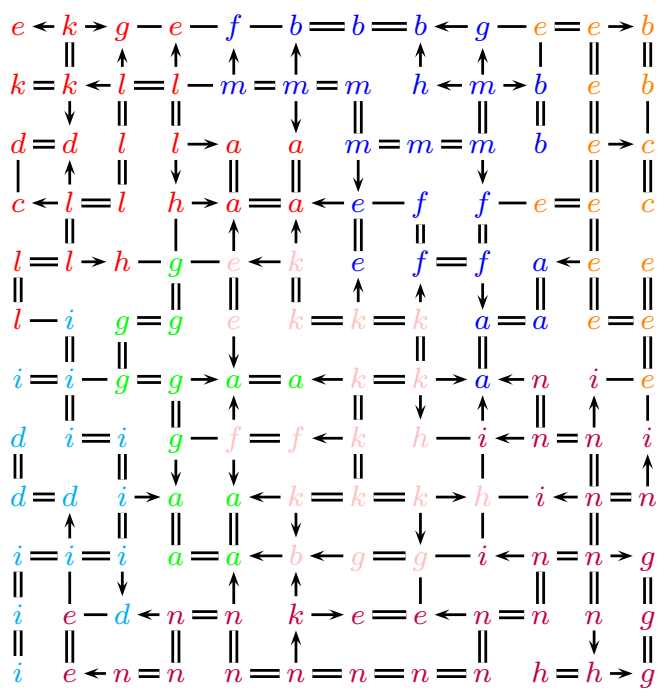

Fig. 10 A partition $\Sigma$ of influence zones for the image $I$ of Figures 3 and 7 . The seven colours correspond to the seven influence zones, where each influence zone is associated to one leaf-point (Figure 8).

Example 12 Figure 10 illustrates a partition of $\Omega$ into seven influence zones induced by each of the seven leafpoints. We use the same colour conventions as in Figure 8. By definition, the influence zone of a leaf-point is a subset of its corresponding reachable zone. In the case of the red leaf-point, the influence zone is the same as its reachable zone (Figure 9). By contrast, in the case of the blue leaf-point, the influence zone is a strict subset of its reachable zone. Indeed, the yellow flat zone of value a belongs to both reachable zones, and we had to arbitrarily choose to include it into one of the two associated influence zones.

Remark 12 Contrary to the cover $P$ of reachable zones, a partition $\Sigma$ of influence zones is not unique, in general, for a given image I. However, this nondeterminism corresponds to overlapping areas in reachable zones (namely, points accessible by a descent paradigm from various leaf-points), and then it will not have any impact on the component-graph construction result. The influence zones can be computed by a concurrent seeded region-growing process, considering $\Lambda$ as set of seeds. The time cost for computing the partition $\Sigma$ is no longer output-dependent, and is linear, namely $\mathcal{O}(|\Omega|)$.

\section{Influence zone graph of an image}

The partition $\Sigma$ subdivides the support $\Omega$ of the image $I$ into $|\Lambda|$ regions $(1 \leq|\Lambda| \leq|\Omega|)$. Each of these regions

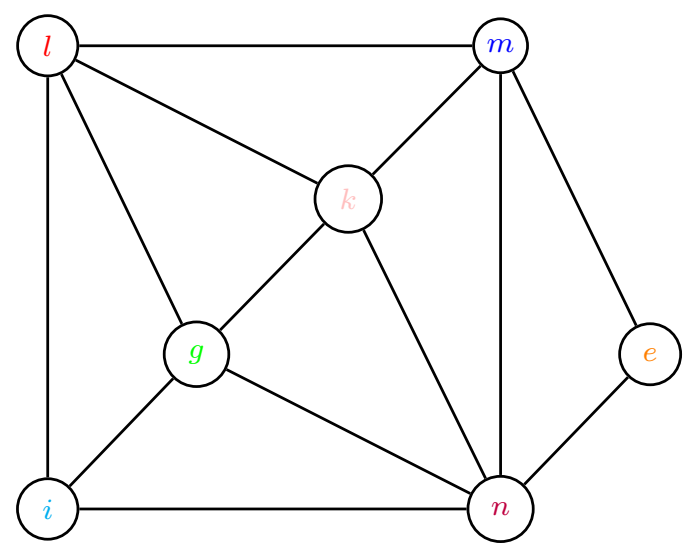

Fig. 11 The influence zone graph $\mathfrak{Z}=(\Lambda, \frown \Lambda)$ associated to the partition $\Sigma$ (Figure 10) of the image $I$ of Figures 3 and 7. Each disk corresponds to a leaf-point $x \in \Lambda$. The value in the disk is the value $I(x)$ of this leaf-point, and the colour indicates the corresponding influence zone in Figure 10. The adjacency $\frown_{\Lambda}$ is represented by edges between the disks.

$\sigma(x)$ contains exactly one leaf-point $x \in \Lambda$ associated to a leaf $L_{x}=(\{x\}, I(x))$ of the component graph $\mathfrak{G}$.

We are now describing the structure of these $|\Lambda|$ regions within the image support. In particular, we are interested by the adjacency relations between them. Indeed, this will allow us to further compute the nodes and their relations in the strong component-graph.

To this end, we define a graph called the influence zone graph, where vertices are the regions $\sigma(x), x \in \Lambda$, and the adjacency relation is induced by the adjacency relation $\frown$ on $\Omega$. Since the mapping $\sigma: \Lambda \rightarrow \Sigma$ is a bijection, we can define, without loss of generality, this adjacency relation, then noted $\frown_{\Lambda}$, on $\Lambda$ instead of $\Sigma$.

Definition 7 (Influence zone graph) The influence zone graph of an image $I$ is the graph $\mathfrak{Z}=\left(\Lambda, \frown_{\Lambda}\right)$ where $\frown_{\Lambda}$ is defined, for any distinct leaf-points $x, y \in \Lambda$ as

$(x \frown \Lambda y) \Leftrightarrow\left(\exists x^{\prime} \in \sigma(x), \exists y^{\prime} \in \sigma(y), x^{\prime} \frown y^{\prime}\right)$

Example 13 Figure 11 illustrates the influence zone graph of the partition $\Sigma$ of I defined in Figure 10. This graph has seven nodes, which correspond to the seven leaf-points in Figure 8 and, equivalently, to the induced seven influence zones in Figure 10. For instance, the upper-left red node, labeled by the value $l$ corresponds to the red leaf-point of value $l$ in the upper-left part of the image, in Figures 8-10. This node is adjacent to four other nodes, labeled by $m$ (in blue), $k$ (in pink), $g$ (in green) and $i$ (in cyan). Indeed, in Figure 10, the red influence zone is adjacent to the blue one via four couples of adjacent points located on the first and second zone, respectively. This is the same for the pink zone (two adjacency links, between two pink points and 
a same unique red point), the green zone (two adjacency links, between two red points and a same unique green point), and the cyan zone (one adjacency link, between a red point of value $l$ and a cyan point of value $i$ ).

Let us consider two distinct leaf-points $x, y \in \Lambda$ such that $x \frown_{\Lambda} y$. There exists a path $x=x_{0} \frown \ldots \frown x_{i}=$ $x^{\prime} \frown y^{\prime}=x_{i+1} \frown \ldots \frown x_{t}=y(0 \leq i<t \geq 1)$ in $\Omega$, such that $S_{x}=\left\{x_{j} \mid j \in \llbracket 0, i \rrbracket\right\} \subseteq \sigma(x)$ and $S_{y}=\left\{x_{j} \mid j \in \llbracket i+1, t \rrbracket\right\} \subseteq \sigma(y)$.

By construction, for all $p \in S_{x}$ (resp. $S_{y}$ ), we have $I(p) \geqslant I\left(x^{\prime}\right)\left(\operatorname{resp} . I(p) \geqslant I\left(y^{\prime}\right)\right)$. Then, for all $p \in$ $S_{x} \cup S_{y}$, and for all $v \in I\left(x^{\prime}\right)^{\downarrow} \cap I\left(y^{\prime}\right)^{\downarrow}$, we have $I(p) \geqslant v$.

This leads us to define a valuation of the edges of $\frown_{\Lambda}$ as follows

$$
\mid \begin{aligned}
\nu: \frown \Lambda & \rightarrow 2^{V} \\
(x, y) & \mapsto \bigcup_{\left(x^{\prime}, y^{\prime}\right) \in E(x, y)} I\left(x^{\prime}\right)^{\downarrow} \cap I\left(y^{\prime}\right)^{\downarrow}
\end{aligned}
$$

where

$E(x, y)=\left\{\left(x^{\prime}, y^{\prime}\right) \mid x^{\prime} \in \sigma(x), y^{\prime} \in \sigma(y), x^{\prime} \frown y^{\prime}\right\}$

The function $\nu$ provides the values $v$ of $V$ that allow us to define sequences of points between two adjacent influence zones, that connect the two associated leafpoints, while remaining below $v$.

More formally, we have the following property.

Property 9 Let $x, y \in \Lambda$ such that $x \frown_{\Lambda} y$. The following two statements are equivalent

(i) $v \in \nu((x, y))$; and

(ii) there exists a sequence $x=x_{0} \frown \ldots \frown x_{t}=y$ $(t \geq 1)$ such that for all $i \in \llbracket 0, t \rrbracket, x_{i} \in \sigma(x) \cup \sigma(y)$ and $I\left(x_{i}\right) \geqslant v$.

Example 14 In the graph of Figure 11, let us consider the red node of value $l$ and the cyan node of value $i$. They correspond to the red and cyan influence zones in Figure 10, respectively. These two nodes are adjacent for the $\frown_{\Lambda}$ relation. Indeed, there exist a point of value $l$ in the red zone and a point of value $i$ in the cyan zone which are adjacent for the $\sim$ relation. In other words, this couple of points belongs to $E(\cdot, \cdot)$ (it is actually the unique such pair). The value of $\nu$ for the corresponding edge between the red and cyan leaf-points is then equal to $l^{\downarrow} \cap i^{\downarrow}=\{a, c, d, f\}$.

Remark 13 It is sufficient to consider the restricted function $\nu \nabla: \frown_{\Lambda} \rightarrow 2^{V}$ defined, for any $x \frown_{\Lambda} y$ as

$$
\begin{aligned}
\nu \nabla((x, y)) & =\nabla^{\leqslant} \nu((x, y)) \\
& =\nabla_{\left(x^{\prime}, y^{\prime}\right) \in E(x, y)}^{\leqslant} \bigcup^{\leqslant} I\left(x^{\prime}\right)^{\downarrow} \cap I\left(y^{\prime}\right)^{\downarrow}
\end{aligned}
$$

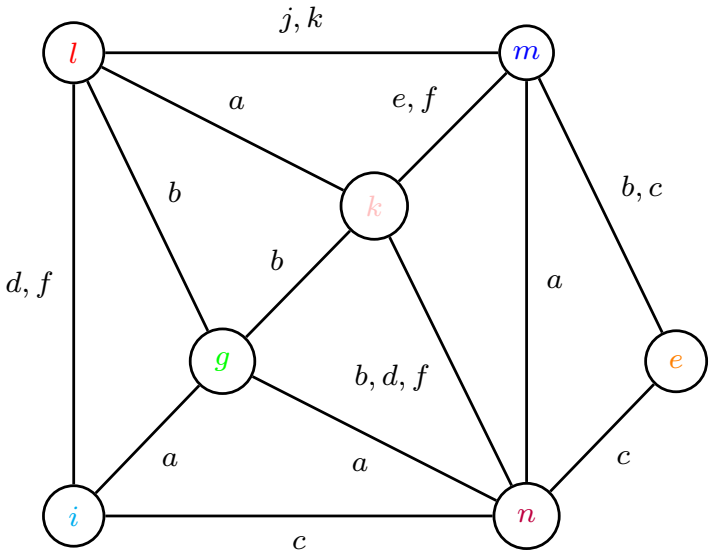

Fig. 12 The influence zone graph $\mathfrak{Z}=\left(\Lambda, \wedge_{\Lambda}\right)$ of Figure 11 is now endowed with the function $\nu \nabla$. For each pair of adjacent leaf-points $x \frown_{\Lambda} y$, the set of values $\nu \nabla((x, y))$ is provided as a valuation of the edge between the two related vertices of $\mathfrak{Z}$. The computation of these sets of values first requires to compute the sets $E(x, y)$ (Equation (40)), and then $\nu \nabla((x, y))$ by following Equation (42).

(In practice, the formulation of Equation (42) is used for actually computing $\nu \nabla((x, y))$.) Using $\nu \nabla$ allows us to reduce the space cost of $\nu$, by only storing the minimal set of values required to identify the paths between the leaf-points $x$ and $y$ within $\sigma(x) \cup \sigma(y)$. In particular, in Property 9, Statement ( $i)$ then rewrites as " $\exists w \in$ $\nu \nabla((x, y)), v \leqslant w$ " or " $v \in \bigcup_{w \in \nu \nabla((x, y))} w^{\downarrow} "$

Example 15 The value of $\nu \nabla$ for the edge between the red and cyan leaves (see previous example) is equal to $\{d, f\}$, since $c \leqslant f$ and $a \leqslant d, f$. Figure 12 illustrates the valuation of the influence zone graph of Figure 11 with the function $\nu \nabla$. For instance, for the red and blue leaf-points, noted $x$ and $y$, of value $l$ and $m$ respectively, the set $E(x, y)$ is composed of four pairs of points, that correspond to the four edges of $\frown$ in Figure 10 linking a red point $x^{\prime}$ and a blue point $y^{\prime}$. The associated four pairs of values $\left(I\left(x^{\prime}\right), I\left(y^{\prime}\right)\right)$ are $(e, f),(l, m),(a, m)$ and $(a, e)$. Then the four sets $\nabla^{\leqslant} I\left(x^{\prime}\right)^{\downarrow} \cap I\left(y^{\prime}\right)^{\downarrow}$ are $\{c\} \quad$ (as $\left.c=\gamma^{\leqslant} e^{\downarrow} \cap f^{\downarrow}\right),\{j, k\}$ (as $\left.\{j, k\}=\nabla^{\leqslant} l^{\downarrow} \cap m^{\downarrow}\right),\{a\}$ and $\{a\}$ (as $\left.a \leqslant e, m\right)$. The union of these four sets is $\bigcup_{\left(x^{\prime}, y^{\prime}\right) \in E\left(x_{l}, x_{m}\right)} \nabla^{\leqslant} I\left(x^{\prime}\right)^{\downarrow} \cap$ $I\left(y^{\prime}\right)^{\downarrow}=\{a, c, j, k\}$ and the set of maximal elements is $\nabla^{\leqslant} \bigcup_{\left(x^{\prime}, y^{\prime}\right) \in E(x, y)} \nabla^{\leqslant} I\left(x^{\prime}\right)^{\downarrow} \cap I\left(y^{\prime}\right)^{\downarrow}=\{j, k\}$ (Equation (42)), since $a \leqslant j, k$ and $c \leqslant j$. We then have $\nu \nabla((x, y))=\{j, k\}$.

Remark 14 From an algorithmic point of view, $\nu \nabla$ can be built independently for any distinct $x \frown_{\Lambda} y$. In particular, for each $x \frown_{\Lambda} y$, the couples of points $\left(x^{\prime}, y^{\prime}\right) \in E(x, y)$ can be gathered during the construction of the influence zones $\sigma(x)$ and $\sigma(y)$, and $\nu \nabla$ can then be built on the flight. 


\section{Component-graph modelling}

10.1 Node modelling

A node $K=(X, v)$ corresponds to a part $X \subseteq \Omega$ of the support of $I$, and a value $v \in V$. By construction, there exists (at least) one leaf $L_{x}=(\{x\}, I(x)) \in \Delta^{\unlhd} \stackrel{\circ}{\Theta}$ such that $L_{x} \unlhd K$. In particular, we have $v \leqslant I(x)$ and $x \in X$. In addition, $K$ is the only node of value $v$ such that $x \in X$. As a consequence, the leaf-point $x$ can be used for identifying $K$. In other words, we can rewrite $K$ as $N(x, v)$, that means "the (only) node of $\stackrel{\mathfrak{G}}{\text { of value }}$ $v$, whose support $X$ contains $x "$.

More formally, we can define the function

$$
\begin{aligned}
N: \Lambda \times V & \rightarrow \Theta \cup\{\alpha\} \\
(x, v) & \mapsto \begin{cases}K=(X, v) \text { with } x \in X & \text { if } v \leqslant I(x) \\
\alpha & \text { otherwise }\end{cases}
\end{aligned}
$$

We set $N(x, v)=\alpha$ whenever $v \notin I(x)$. This is a convention that allows us to make $N$ fully defined on $\Lambda \times V$ despite the fact that all the couples $(x, v)$ are not necessarily associated to a node of the component-graph.

The function $N$ enables to identify all the nodes of a component-graph $\mathfrak{G}$. In other words, $N$ is surjective (note that if $N^{-1}(\{\alpha\})=\emptyset$, we can omit $\alpha$ in the definition of $N$, and the surjectivity is still preserved). However, a node $K=(X, v)$ may be associated to many couples $(x, v)$, i.e. many leaf-points $x$ can belong to $X$. In other words, $N$ may be non-injective.

In order to tackle this issue, we assign a numerical label to each leaf-point. We define a bijective function

$$
\begin{aligned}
\ell: \Lambda & \rightarrow \llbracket 1,|\Lambda| \rrbracket \subset \mathbb{N} \\
x & \mapsto \ell(x)
\end{aligned}
$$

We note $\ell_{x}=\ell(x)$ the label of the leaf-point $x$. If $x$ has a label $\ell(x)=k$, it is also noted $x_{k}$.

Then, we can define, for any $K=(X, v) \in \Theta$ a canonical leaf-point $x$ for $K$, noted $x_{K}$. It is defined as the leaf-point $x \in X$ of minimal label, that is

$x_{K}=\arg _{\Lambda} \min \{\ell(x) \mid x \in X\}$

The label of this canonical leaf-point $x_{K}$ is noted $\ell_{K}$. The node $K$ is then characterized by the couple $\left(\ell_{K}, v\right)$.

Remark 15 A leaf $L_{x}=(\{x\}, I(x)) \in \Delta^{\unlhd} \Theta$ is necessarily characterized by the couple $\left(\ell_{x}, I(x)\right)$.

Example 16 In Figure 13, we provide a specific label $\ell$ for each leaf-point of the influence zone graph of Figures 11 and 12. In particular, we set the label 1 to the red leaf-point, 2 to the blue, 3 to the pink, 4 to the green, 5 to the purple, 6 to the cyan and 7 to the orange. The obtained couples $(\ell, v)$ then correspond to the corresponding leaves of the associated component-graph.

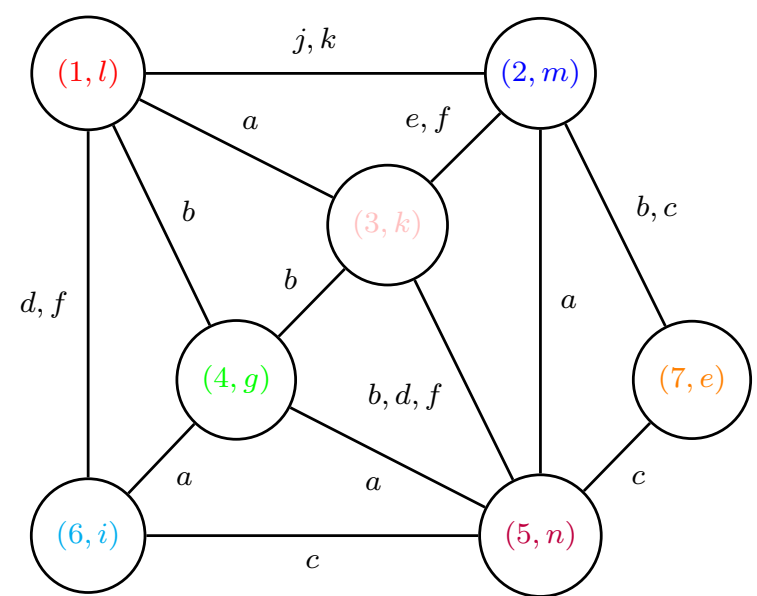

Fig. 13 The influence zone graph $\mathfrak{Z}=(\Lambda, \frown \Lambda)$ of Figures 11 and 12 , where each leaf-point $x$ of value $I(x)=v$ has now a specific label $\ell$. Each couple $(\ell, v)$ then characterizes one of the seven leaves of the component-graph $\mathfrak{G}$ of the image $I$ of Figures 3 and 7 .

Property 10 For any $K_{1}, K_{2} \in \Theta$, we have

$\left(K_{1} \unlhd K_{2}\right) \Rightarrow\left(\ell_{K_{2}} \leq \ell_{K_{1}}\right)$

In particular we have $\ell_{(\Omega, \perp)}=1$.

Remark 16 From now on, we will note indistinctly a node $K=(X, v)$ as $K$ or $\left(\ell_{K}, v\right)$.

From a structural point of view, building the set of nodes $\stackrel{\ominus}{\Theta}$ of a component-graph $\stackrel{\circ}{G}$ is then equivalent to determining the subset of all the couples $\left(\ell_{K}, v\right)$ within $\llbracket 1,|\Lambda| \rrbracket \times V$ that characterize these nodes.

\subsection{Edge modelling}

Until now, the discussions were valid for both strong and weak component-graphs.

At this stage, we now focus on the strong component-graph $\mathfrak{G}_{s}$, and especially on $\mathfrak{G}_{s}$, that contains all the nodes of $\Theta$. In the next sections, we will aim at building $\mathfrak{G}_{s}$.

The strong component-graph $\mathfrak{G}_{s}$ is the Hasse dia$\operatorname{gram}\left(\Theta, \triangleleft_{s}\right)$ of the ordered set $\left(\Theta, \unlhd_{s}\right)$. From the very definition of $\unlhd_{s}$, we have the following property.

Property 11 Let $K_{1}=\left(X_{1}, v_{1}\right), K_{2}=\left(X_{2}, v_{2}\right) \in \Theta$. We have

$\left(K_{1} \boldsymbol{\triangleleft}_{s} K_{2}\right) \Rightarrow\left(v_{2} \prec v_{1}\right)$

This equation rewrites as

$\left(\left(\ell_{K_{1}}, v_{1}\right) \triangleleft_{s}\left(\ell_{K_{2}}, v_{2}\right)\right) \Rightarrow\left(v_{2} \prec v_{1}\right)$

In other words, an edge of $\mathfrak{G}_{s}$ between $K_{1}$ and $K_{2}$ can be modelled by the couple of labels $\left(\ell_{K_{2}}, \ell_{K_{1}}\right)$ associated to the edge $\left(v_{2}, v_{1}\right)$ of $(V, \prec)$. 
10.3 Strong component-graph modelling

We now show how the strong component-graph $\mathfrak{G}_{s}$ can be modelled - and then stored - by enriching the Hasse diagram $(V, \prec)$.

First, we observed that a node $K=(X, v)$ can be characterized as $\left(\ell_{K}, v\right)$, where $\ell_{K} \in \llbracket 1,|\Lambda| \rrbracket \subset \mathbb{N}$ is the minimal label associated to the canonical leaf-point of $K$. Consequently, we can define the function

$$
\mid \begin{aligned}
\theta: V & \rightarrow 2^{\llbracket 1,|\Lambda| \rrbracket} \\
v & \mapsto\left\{\ell_{K} \mid K=(X, v) \in \Theta\right\}
\end{aligned}
$$

In other words, $\theta$ provides, for each value of $V$, the set of all the canonical labels characterizing the nodes defined at this value.

Second, we observed that an edge between two nodes $K_{1}=\left(\ell_{K_{1}}, v_{1}\right)$ and $K_{2}=\left(\ell_{K_{2}}, v_{2}\right)$ can be modelled as the couple $\left(\ell_{K_{2}}, \ell_{K_{1}}\right)$ associated to the edge between $v_{2}$ and $v_{1}$ in $(V, \prec)$. Consequently, we can define the function

$$
\begin{aligned}
\varepsilon: \prec & \rightarrow 2^{\llbracket 1,|\Lambda| \rrbracket \times \llbracket 1,|\Lambda| \rrbracket} \\
\left(v_{2}, v_{1}\right) & \mapsto\left\{\left(\ell_{K_{2}}, \ell_{K_{1}}\right) \mid\left(\ell_{K_{1}}, v_{1}\right) \varangle_{s}\left(\ell_{K_{2}}, v_{2}\right)\right\}
\end{aligned}
$$

In other words, $\varepsilon$ provides, for each edge of the Hasse diagram of $(V, \leqslant)$ between two successive values, the set of all the pairs of canonical labels corresponding to successive nodes associated to these two values, respectively, in the component graph.

The information carried by the enriched Hasse diagram $(V, \prec, \theta, \varepsilon)$ is sufficient for modelling the strong component-graph $\mathfrak{G}_{s}=\left(\Theta, \boldsymbol{\varangle}_{s}\right)$. This is formalized by the following proposition, that directly derives from the above definitions and properties.

Proposition 4 Let $\mathfrak{G}_{s}$ be the strong component-graph of an image $I$, and $(V, \prec, \theta, \varepsilon)$ the associated enriched Hasse diagram. Let $K=(X, v), K_{1}=\left(X_{1}, v_{1}\right), K_{2}=$ $\left(X_{2}, v_{2}\right)$. We have:

$$
\begin{aligned}
(K \in \Theta) & \Leftrightarrow\left(\ell_{K} \in \theta(v)\right) \\
\left(K_{1} \varangle_{s} K_{2}\right) & \Leftrightarrow\left(\left(\ell_{K_{2}}, \ell_{K_{1}}\right) \in \varepsilon\left(\left(v_{2}, v_{1}\right)\right)\right)
\end{aligned}
$$

Example 17 The enriched Hasse diagram $(V, \prec, \theta, \varepsilon)$ associated to the strong component-graph $\mathfrak{G}_{s}$ of Figure 5 is illustrated in Figure 14. Let us focus, for instance, on the value $k$. We observe that $\theta(k)=\{1,3,5\}$. This means that there are three nodes of $\Theta$ at value $k$; they correspond to the nodes labeled $(1, k),(3, k)$ and $(5, k)$ in Figure 5. Regarding the nodes lower than these three nodes, we have $\varepsilon((k, l))=\{(1,1)\}, \varepsilon((k, m))=\{(1,2)\}$ and $\varepsilon((k, n))=\{(5,5)\}$. This means that we have $(1, l)$ $\boldsymbol{\varangle}_{s}(1, k),(2, m)$

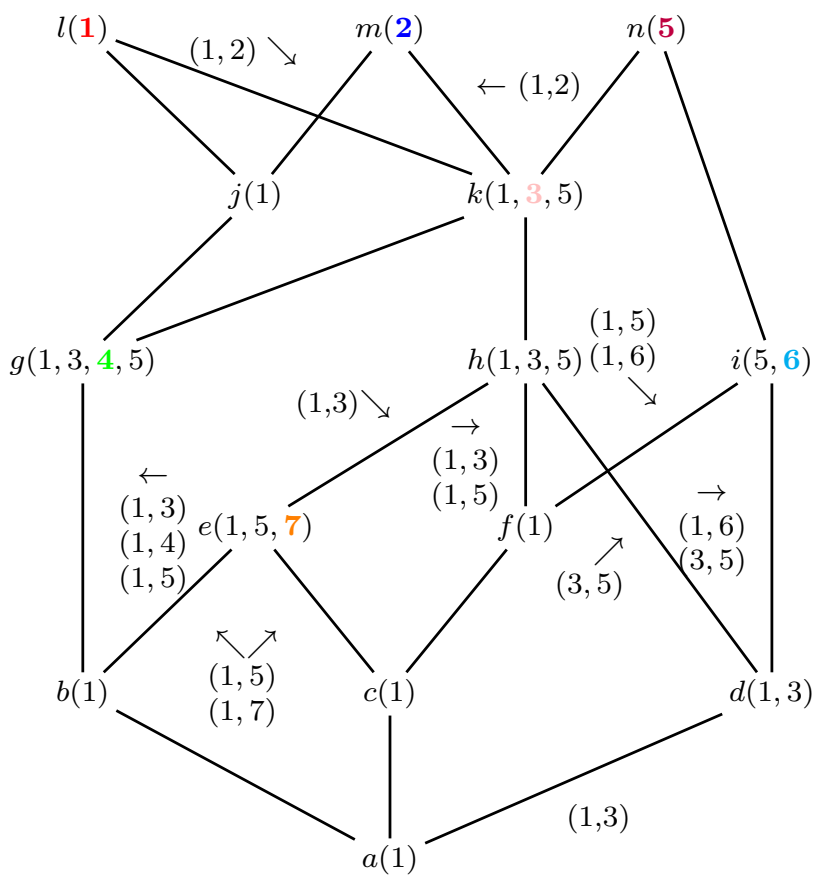

Fig. 14 The strong component-graph $\mathfrak{G}_{s}$ of the image $I$ of Figures 3 and 7 . This component-graph is the same as in Figure 5. However, it is modelled here as the Hasse diagram of $(V, \leqslant)$ (Figure 2 ) enriched with the functions $\theta$ and $\varepsilon$. For each value $v \in V$, the labels of the set $\theta(v)$ are provided into brackets. For each couple of adjacent values $v \prec w$, the elements of $\varepsilon((v, w))$ are provided as a valuation of the edge between $v$ and $w$. Note that when a couple $(x, x)$ belongs to $\varepsilon((v, w))$, it is useless to represent it, as we necessarily have $x \in \theta(v)$ and $x \in \theta(w)$. The labels corresponding to leaves are provided in bold and colour fonts (the colours correspond to those used in Figures 8-13).

There is no node lower than $(3, k)$, which means that it is a leaf of $\mathfrak{G}_{s}$ (Figure 5). Regarding the nodes greater than these three nodes, we have $\varepsilon((g, k))=$ $\varepsilon((h, k))=\{(1,1),(3,3),(5,5)\}$. This means that we have $(1, k) \boldsymbol{\triangleleft}_{s}(1, g),(1, h) ;(3, k) \boldsymbol{\triangleleft}_{s}(3, g),(3, h) ;$ and $(5, k) \boldsymbol{\triangleleft}_{s}(5, g),(5, h)$ (Figure 5$)$. Note that when a couple $(x, x)$ belongs to $\varepsilon((v, w))$, it is useless to represent it, as we necessarily have $x \in \theta(v)$ and $x \in \theta(w)$. In particular, in the case of the edge between $g$ (resp. $h$ ) and $k$, no information has to be explicitly stored in the enriched Hasse diagram (Figure 14).

In the next section, we explain how to build $\theta$ and $\varepsilon$, and then the strong component-graph $\mathfrak{G}_{s}$.

\section{Strong component-graph computation}

\subsection{Connected component computation}

A node $K=(X, v)$ is defined by its support $X$ and its value $v$. Its support $X$ contains one or many leaf- 
point(s) $x$. In particular, $X$ is subdivided by the influence zones of these leaf-points, and this subdivision forms a partition

$X=\bigsqcup_{x \in \Lambda \cap X} \rho(x) \cap \lambda_{v}(I)$

In other words, $X$ can be retrieved from the set of the leaf-points it contains.

By definition, all the leaf-points in the support of a node are connected within the thresholded influence zone graph $\mathfrak{Z}^{v}=\left(\Lambda^{v}, \frown_{\Lambda}^{v}\right)$ obtained at value $v$, defined by

$\Lambda^{v}=\{x \in \Lambda \mid I(x) \geqslant v\}$

$\frown_{\Lambda}^{v}=\{(x, y) \mid v \in \nu((x, y))\}$

In particular, building the nodes $K=(X, v)$ at a given value $v \in V$ consists of building the connected components of $\mathfrak{Z}^{v}=\left(\Lambda^{v}, \frown_{\Lambda}^{v}\right)$.

The connectedness relation $\leftrightarrow_{\Lambda}^{v}$ is the reflexivetransitive closure of the adjacency relation $\frown_{\Lambda}^{v}$. It is convenient to model adjacency and connectedness relations in a Boolean matrix form. Indeed, this will allow us to simply express the connected component computation (namely, a transitive closure problem) as a matrix product procedure.

Let us consider the Boolean square, symmetric matrix $A_{v}$ of dimension $|\Lambda| \times|\Lambda|$, defined as $A_{v}=$ $\left[a_{i, j}^{v}\right]_{1 \leq i, j \leq|\Lambda|}$ with

$a_{i, j}^{v}= \begin{cases}1 & \text { if } x_{i} \frown_{\Lambda}^{v} x_{j} \\ 1 & \text { if } i=j \\ 0 & \text { otherwise }\end{cases}$

where $\Lambda=\left\{x_{i}|i \in \llbracket 1,| \Lambda \mid \rrbracket\right\}$. This matrix models the reflexive closure of the adjacency relation $\frown_{\Lambda}^{v}$.

The induced connectedness relation - that defines the connected components of leaf-points, and thus the supports of the nodes at value $v$-is modelled by the Boolean square, symmetric matrix $C_{v}$ of dimension $|\Lambda| \times|\Lambda|$, defined as $C_{v}=\left[c_{i, j}^{v}\right]_{1 \leq i, j \leq|\Lambda|}$ with

$c_{i, j}^{v}= \begin{cases}1 & \text { if } x_{i} \leftrightarrow_{\Lambda}^{v} x_{j} \\ 0 & \text { otherwise }\end{cases}$

and we compute $C_{v}$ as

$C_{v}=\lim _{n} A_{v}^{n}$

The time complexity for computing $C_{v}$ is $\mathcal{O}\left(|\Lambda|^{3}\right)$, that is the standard time complexity for the transitive closure of a graph.
Remark 17 The matrix $A_{v}$ is sparse. In particular, it has empty rows and columns for all the labels $k$ such that $a_{k, k}=0$. These labels $k$ correspond to leaf-points $x_{k}$ such that $I\left(x_{k}\right) \ngtr v$. This allows us to reduce the $|\Lambda|$ term to $|\{x \in \Lambda \mid I(x) \geqslant v\}|$ in $\mathcal{O}\left(|\Lambda|^{3}\right)$.

Remark 18 The matrix $A_{v}$ is Boolean. As a consequence, the matrix product involves Boolean operators $\wedge$ and $\vee$ instead of . and + . Consequently, the result of the calculus of an element is 1 as soon as one of the operands of the $\vee$ combination between the $|\Lambda|$ binary terms in $\wedge$ is 1 . This also allows us to reduce the time cost of the overall computation by avoiding useless elementary operations.

The time complexity of the $C_{v}$ computation can be optimized by considering the structure of the $\unlhd_{s}$ relation, that is directly linked to that of $\leqslant$.

Property 12 Let $v, w \in V$. Let us assume that $v \leqslant w$. Let $Y$ be a connected component of $\mathfrak{Z}^{w}$. Then, there exists a connected component $X$ of $\mathfrak{Z}^{v}$ such that $Y \subseteq X$.

The next result is an immediate corollary.

Corollary 1 Let $v, w \in V$. Let us assume that $v \prec w$. Let $K^{\prime}=(Y, w) \in \Theta$ be a node of $\mathfrak{G}_{s}$. Then, there exists a node $K=(X, v) \in \Theta$ such that $K^{\prime} \varangle_{s} K$.

As a consequence, for any $v \in V$, the computation of $C_{v}$-which is done from $A_{v}$, in theory - can be carried out recursively by determining the transitive closure of the matrix $B_{v}=\left[b_{i, j}^{v}\right]_{1 \leq i, j \leq|\Lambda|}$ of dimension $|\Lambda| \times|\Lambda|$ defined as follows

$b_{i, j}^{v}=\bigvee\left\{\begin{array}{l}\bigvee_{v \prec w} c_{i, j}^{w} \\ v \in \nu \nabla\left(\left(x_{i}, x_{j}\right)\right) \\ \left(i=j \wedge I\left(x_{i}\right)=v\right)\end{array}\right.$

The computation of $B_{v}$ simply consists of combining the connected component matrices at the directly greater values, and then adding two kinds of information: on the one hand, the adjacency links at value $v$ between pairs of leaf-points (these links justify a posteriori the definition of the influence zone graph); on the other hand the reflexive links associated to the leafpoints that correspond to leaves at value $v$.

Then, we compute $C_{v}$ as

$C_{v}=\lim _{n} B_{v}^{n}$

This computation converges more rapidly than the transitive closure of $A_{v}$, since a part of the calculus was already carried out in the $C_{w}$ matrices $(v \prec w)$. 
Remark 19 In $B_{v}$, the lines and columns $k$ such that $x_{k, k}$ is a leaf-point that satisfies $I\left(x_{k, k}\right)=v$ necessarily contain 0 values everywhere but in $(k, k)$. As a consequence, these rows and columns can be omitted in the computation of $B_{v}$, and updated only after the transitive closure calculus. This allows to reduce the overall time complexity for building $C_{v}$.

Example 18 An example of whole computation of the connected components of the thresholded influence zone graphs $\mathfrak{Z}^{v}$ associated to the influence zone graph $\mathfrak{Z}$ of Figure 13 is proposed in Appendix C. In the same appendix, we also derive from these results the $\theta$ and $\varepsilon$ functions that allow us to model the strong componentgraph $\mathfrak{G}_{s}$ of the image I of Figures 3 and 7.

\subsection{Building the nodes of the component-graph}

Let $v \in V$, and let us suppose that the matrix $C_{v}$ has been computed. Each non-empty row of $C_{v}$ corresponds to a given node of $\Theta$ at value $v$. More precisely, for the row $k \in \llbracket 1,|\Lambda| \rrbracket$, this node is $K=(X, v)$ where

$$
X=\bigcup_{\substack{1 \leq i \leq|\Lambda| \\ c_{(k, i)}^{v} \neq 0}} \rho\left(x_{i}\right) \cap \lambda_{v}(I)
$$

Practically, any node $K$ can be modelled by its canonical label $\ell_{K}$. Each node is then identified by the $\ell_{K}$-th row. Any such row is empty in the lower triangular matrix, that is

$\ell_{K}=\min \left\{i \in \llbracket 1,|\Lambda| \rrbracket \mid c_{\left(\ell_{K}, i\right)}^{v}=1\right\}$

Determining the nodes of $\Theta$ at value $v$ is then simply done by scanning the lines of $C_{v}$. More precisely, this research can be restricted to the rows that already correspond to canonical labels of nodes at values $w \in V$ with $w \prec v$ plus, of course, the labels $\ell_{x}$ of leaf-points $x$ that satisfy $I(x)=v$, which correspond to leaves $(\{x\}, I(x))$.

Following the definition of the function $\theta$ (Equation (49)), we finally define the set of nodes of $\Theta$ at value $v$ as

$\theta(v)=\left\{k \in \llbracket 1,|\Lambda| \rrbracket \mid\left(1-c_{(k, k)}^{v}\right) \vee \bigvee_{i=1}^{k-1} c_{(k, i)}^{v}=0\right\}$

Example 19 In the matrix $C_{e}$ (Equation (78), in $A p$ pendix $C)$, we have $1 \in \theta(e)$, since $1-c_{(1,1)}^{e}=1-1=0$. However, we have $2 \notin \theta(e)$, since $c_{(2,1)}^{e}=1$. We also have $4 \notin \theta(e)$, since $1-c_{(4,4)}^{e}=1-0=1$. Finally, we have $5 \in \theta(e)$, since $1-c_{(5,5)}^{e}=1-1=0$ whereas $c_{(5, i)}^{e}=0$ for $i=1, \ldots, 4$.
Remark 20 The identification of the nodes of $\ddot{\Theta}$ can be made by scanning, for each node $K=(X, v)$ of label $\ell_{K}$, if there exists a leaf-point $x \in X$ (i.e., such that $\left.c_{\left(\ell_{K}, \ell_{x}\right)}^{v}=1\right)$ with a point $y \in \rho(x)$ such that $I(y)=v$.

11.3 Building the edges of the component-graph

Let $K^{\prime}=(Y, w)$ be a node of $\Theta$ at value $w$, and let us suppose that we have computed the set $\theta(v)$ of the nodes at value $v$ with $v \prec w$ and the associated matrix $C_{v}$.

There is exactly one node $K=(X, v)$ such that $K^{\prime} \boldsymbol{\iota}_{s} K$. By construction of $C_{v}$, the canonical label $\ell_{K}$ of this node is defined as

$\ell_{K}=\min \left\{i \in \llbracket 1, \Lambda \rrbracket \mid c_{\left(\ell_{K^{\prime}}, i\right)}^{v}=1\right\}$

Then, following the definition of the function $\varepsilon$ (Equation (50)), we define the set of edges of $\boldsymbol{\triangleleft}_{s}$ between the values $v \prec w$ as

$\varepsilon((v, w))=\left\{\left(\ell_{x}, \ell_{y}\right) \mid \ell_{y} \in \theta(w), \ell_{x}=\min _{i}\left\{c_{\left(\ell_{y}, i\right)}^{v}=1\right\}\right\}$

Example 20 Let us consider the function $\varepsilon$ for the couple of values $(d, i)$. From the matrix $C_{i}$ (Equation (71), in Appendix $C)$, we know that $\theta(i)=\{5,6\}$, whereas from the matrix $C_{d}$ (Equation (80), in Appendix $C$ ), we know that $\theta(d)=\{1,3\}$. From the 5 th row of matrix $C_{d}$, we have $3=\min _{i}\left\{c_{(5, i)}^{d}=1\right\}$, and from the 6th row of matrix $C_{d}$, we have $1=\min _{i}\left\{c_{(6, i)}^{d}=1\right\}$. It follows that $\varepsilon((d, i))=\{(1,6),(3,5)\}$.

\section{Data structure}

Finally, the strong component-graph $\mathfrak{G}_{s}$ can then be modelled and stored as the enriched Hasse diagram $(V, \prec, \theta, \varepsilon)$. The space costs of $\theta$ and $\varepsilon$ are the same as that of $\Theta$ and $\boldsymbol{\triangleleft}_{s}$, respectively, since each node is modelled by its canonical label, whereas each edge is modelled by a couple of such labels. The overall cost of the induced data structure is then $\mathcal{O}\left(|V|+|\prec|+|\Theta|+\left|\boldsymbol{\iota}_{s}\right|\right)$, which is generally equal to $\mathcal{O}\left(\left|\boldsymbol{\iota}_{s}\right|\right)$.

Figure 14 illustrates the strong component-graph $\mathfrak{G}_{s}$ of the image $I$ of Figures 3 and 7, in this paradigm of enriched Hasse diagram.

Such data structure describes the nodes from a symbolic point of view, i.e. by their canonical labels. This allows one to carry out a structural analysis of the component-graph, for instance by observing the modifications of topology between the nodes. (By analogy 
with the component-tree, this would correspond to observing the bifurcations between branches, and the endings of the branches.)

However, since a node $K=(X, v)$ is stored as $K=\left(\ell_{K}, v\right)$, we do not have a direct access to $X$. The information $X$ can be retrieved by various ways:

- by first determining all the leaf-points $x \in X$, and then reconstructing $X$ by a seeded-region growing in $\lambda_{v}(I)$; or

- by directly collecting the points $x \in X$ that need then to be stored in $\mathfrak{G}_{s}$.

The second option requires to store, at each node $K$ not only its canonical label $\ell_{K}$, but also all the points $x \in X$ such that $I(x)=v$. This induces an extra space cost of $\mathcal{O}(\Omega)$. In addition the time cost for building one $X$ is then $\mathcal{O}\left(\left|K^{\downarrow}\right|\right)$, since all the nodes below $K$ have to be scanned for collecting the points $x$ at each value greater than $v$.

The first option requires either to store all the labels of the leaf-points $x \in X$, or to retrieve them by scanning the nodes below $K$. In the first case, the overall extra space cost is $\mathcal{O}\left(\sum_{x \in \Lambda} L_{x}^{\uparrow}\right)$, and the time cost for building one $X$ is $\mathcal{O}(|X|)$. In the second case, there is no extra space cost, but the time cost for building $X$ is $\mathcal{O}\left(|X|+\left|K^{\downarrow}\right|\right)$.

Remark 21 The computation of the supports $X$ of the nodes may also be carried out on the flight during the processing of the component-graph, in the cases where such processing requires to scan the data structure in a bottom-up fashion, i.e. from the leaves up to the root.

\section{Conclusion}

In this article, we explained how to build the component-graph of an image taking its values in any (totally or partially) ordered set. In particular, we developed strategies for reducing as much as possible the space cost of the processed data, by considering first a flat zone model of the image, and second an influence zone model based on the local maxima of the image. This led to the symbolic representation of the image as an influence zone graph. Then, we observed that the strong component-graphs have structural regularity properties, compared to the Hasse diagram of the considered ordered value set. This regularity allowed us to develop a recursive strategy for building the connected components at each value. Indeed, this step of connected component construction is the most costly (with a polynomial complexity). Then, reducing both the size of the input (thanks to the notion of influence zone graph) and the time cost (thanks to incremental computation of the connected components and efficient calculus on Boolean matrices) is a relevant way to decrease the time complexity of this crucial step. Finally, we also emphasized how a component-graph could be modelled and stored by enriching the Hasse diagram of the associated ordered set of values.

Building component-graphs in a simple and efficient fashion opens the way to their actual involvement in image processing and analysis procedures. In particular, our next works will consist of extending the standard attribute-based antiextensive filtering process [2, 21 initially developed for component-trees, in the case of grey-level images, in order to make it tractable with component-graphs, in the case of multivalued images.

\section{Acknowledgements}

The research leading to these results was funded by the French Agence Nationale de la Recherche (Grant agreements ANR-15-CE23-0009 and ANR-18-CE45-0018).

\section{A Proofs of propositions}

Proposition 1 We assume that $(V, \leqslant)$ is totally ordered. The component-tree is the Hasse diagram of $(\Psi, \subseteq)$, whereas the (strong and weak) component-graphs $\dot{\mathfrak{G}}$ and $\ddot{\mathfrak{G}}$ are the Hasse diagrams of $(\dot{\Theta}, \unlhd)$ and $(\ddot{\Theta}, \unlhd)$, respectively. Proving the isomorphism between the Hasse diagrams is indeed the same as proving the isomorphism between the ordered sets. Let $X \in \Psi$. There exists a value $v \in V$ such that $X \in \mathcal{C}\left[\lambda_{v}(I)\right]$. We choose $v$ as the maximal value of $V$ satisfying this property (such maximum exists, as $\leqslant$ is total). It is plain that $(X, v) \in \dot{\Theta}$. As $v$ is the maximal value such that $X \in \mathcal{C}\left[\lambda_{v}(I)\right]$, it follows that for any $w>v$, we have $X \nsubseteq \lambda_{w}(I)$, and more generally, $X \nsubseteq \bigcup_{w>v} \lambda_{w}(I)$. Then,

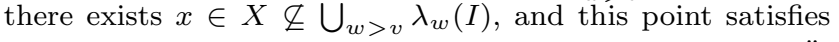
$I(x)=v$. It follows from Equation $(18)$ that $(X, v) \in \ddot{\Theta}$. Let us now assume that $(X, v) \in \ddot{\Theta}$. From Equation (19), we have $(X, v) \in \dot{\Theta}$. Finally, let us assume that $(X, v) \in \dot{\Theta}$. From Equation (17), we directly have $X \in \Psi$. In other words, the projective mapping $\pi=(X, v) \rightarrow X$ between $\dot{\Theta}, \ddot{\Theta}$ and $\Psi$ is a bijection, whereas $\dot{\Theta}=\ddot{\Theta}$. At this stage, since the orders $\unlhd_{s}$ and $\unlhd_{w}$ are the same for $\dot{\Theta}$ and $\ddot{\Theta}$ it is plain that $\left(\dot{\Theta}, \unlhd_{s}\right)$ and $\left(\ddot{\Theta}, \unlhd_{s}\right)$ (resp. $\left(\dot{\Theta}, \unlhd_{w}\right)$ and $\left.\left(\ddot{\Theta}, \unlhd_{w}\right)\right)$ are isomorphic. More strongly, we have $\unlhd_{s}=\unlhd_{w}$. Indeed, for two nodes $K=(X, v), K^{\prime}=(Y, w) \in \dot{\Theta}$, we have $X \subset Y \Rightarrow w \leqslant v$ due to the totality of $\leqslant$. Now, let us consider that $K=(X, v), K^{\prime}=(Y, w) \in \dot{\Theta}$ and $K \unlhd K^{\prime}$. From Equation (8), we have $X \subseteq Y$. Conversely, let us consider that $X, Y \in \Psi$ and $X \subseteq Y$. By considering the inverse function $\pi^{-1}$, and by setting $K=(X, v)=\pi^{-1}(X)$ and $K^{\prime}=(Y, w)=\pi^{-1}(Y)$, we have, by construction, $w \leqslant v$, and it follows that $K \unlhd K^{\prime}$. Finally, the three ordered sets are then isomorphic, and so are the associated Hasse diagrams, namely the component-tree $\mathfrak{T}$ and the two component-graphs $\dot{\mathfrak{G}}$ and $\ddot{\mathfrak{G}}$. $\square$

Proposition 2 Let $x \in \Omega$. Let $X \in \mathcal{C}\left[\lambda_{I(x)}(I)\right]$ be such that

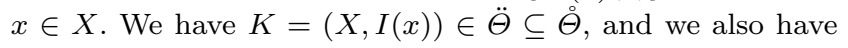




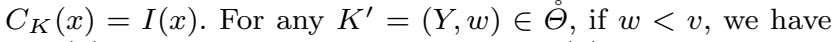
$C_{K^{\prime}}(x) \leqslant w<v$; if $w>v$, we have $C_{K^{\prime}}(x)=\perp<v$; and if $w=v$, we have $C_{K^{\prime}}(x) \leq v$. It follows that $I=\bigvee_{K \in \Theta}^{\leq} C_{K}$.

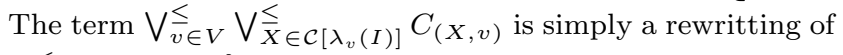
$\bigvee_{K \in \Theta}^{\leq} C_{K}$ for $\stackrel{\Theta}{\Theta}=\Theta$.

Proposition 3 Let $X, Y \in \Theta$. From the definition of $\phi$, we have $X \subseteq Y \Leftrightarrow \phi(X) \subseteq \phi(Y)$. Then, it follows from the definition of $\unlhd_{w}$ and $\unlhd_{s}$ (simply noted $\unlhd$ hereinafter), that $((X, v) \unlhd(Y, w)) \Leftrightarrow\left((\phi(X), v) \unlhd_{\Phi}(\phi(Y), w)\right)$. This is a fortiori true for the transitive reductions 4 and $\boldsymbol{\iota}_{\Phi}$ of $\unlhd$ and $\unlhd_{\Phi}$, respectively. Still from the definition of $\phi$, we have $\phi(\ddot{\Theta})=\ddot{\Theta}_{\Phi}$, and finally, the isomorphism between $(\Theta, \unlhd)$ and $\left(\Theta_{\Phi}, \unlhd_{\Phi}\right)$ also implies that $\phi(\dot{\Theta})=\dot{\Theta}_{\Phi}$. As a consequence, Equation (34) holds in any cases. $\square$

Proposition 4 Equation (51) is a rewritting of the definition of $\theta$ (Equation (49)). Equation (52) is a rewritting of the definition of $\varepsilon$ (Equation (50)).

\section{B Proofs of properties}

Property 1 Equation (16) is simply a rewritting of a part of Equation (12) with $\unlhd_{w}=\unlhd_{2}$ and $\unlhd_{s}=\unlhd_{3}$. What we aim to prove is then the part $K \unlhd_{3} K^{\prime} \Rightarrow K \unlhd_{2} K^{\prime}$ of Equation (12). We set $K=(X, v)$ and $K^{\prime}=(Y, w)$. From Equations (8-9), we have $K \unlhd_{3} K^{\prime} \Leftrightarrow(X, v) \unlhd_{3}(Y, w) \Leftrightarrow X \subseteq Y \wedge w \leqslant v \Leftrightarrow$ $(X \subset Y \vee X=Y) \wedge w \leqslant v \Leftrightarrow(X \subset Y \wedge w \leqslant v) \vee(X=$ $Y \wedge w \leqslant v) \Rightarrow(X \subset Y) \vee(X=Y \wedge w \leqslant v) \Leftrightarrow(X, v) \unlhd_{2}$ $(Y, w) \Leftrightarrow K \unlhd_{2} K^{\prime}$.

Property 2 Let $w \in v^{\downarrow}$. We have $w \leqslant v$, and then $X \subseteq \lambda_{w}(I)$. As $X$ is connected in $\Omega$, it belongs to a unique connected component $X^{\prime} \in \mathcal{C}\left[\lambda_{w}(I)\right]$. In particular, we have $X \subseteq X^{\prime}$ and then $\left(X^{\prime}, w\right) \in K^{\uparrow}$. Thus, $\sigma$ is surjective. Let $\left(Y_{1}, w_{1}\right),\left(Y_{2}, w_{2}\right) \in K^{\uparrow}$ be such that $\sigma\left(\left(Y_{1}, w_{1}\right)\right)=\sigma\left(\left(Y_{2}, w_{2}\right)\right)$. Then, we have $w_{1}=w_{2}$. It follows that $\left(Y_{1}, w_{1}\right),\left(Y_{2}, w_{2}\right) \in \mathcal{C}\left[\lambda_{w_{1}}(I)\right]$. But we have $X \subseteq Y_{1}$ and $X \subseteq Y_{2}$ with $X$ nonempty. Then, we have $Y_{1} \cap Y_{2} \neq \emptyset$, which implies $Y_{1}=Y_{2}$. Thus, $\sigma$ is injective. The result follows. $\square$

Property 3 We set $K_{1}=\left(X_{1}, v_{1}\right)$ and $K_{2}=\left(X_{2}, v_{2}\right)$. We have $K \unlhd_{w} K_{1}$ (resp. $\left.K \unlhd_{w} K_{2}\right)$ and then $X \subseteq X_{1}$ (resp. $\left.X \subseteq X_{2}\right)$. Then, we have $X_{1} \cap X_{2} \neq \emptyset$. Since $X_{1} \in \mathcal{C}\left[\lambda_{v_{1}}(I)\right]$ and $X_{2} \in \mathcal{C}\left[\lambda_{v_{2}}(I)\right]$, the connectedness of $X_{1}$ and $X_{2}$, together with the fact that $v_{1} \geqslant v_{2}$ leads to $X_{1} \subseteq X_{2}$. Thus, we have $\left(X_{1} \subseteq X_{2}\right) \wedge\left(v_{2} \leqslant v_{1}\right)$, i.e. $K_{1} \unlhd_{s} K_{2}$, and a fortiori $K_{1} \unlhd_{w} K_{2}$.

\section{Property 4}

We set $K_{1}=\left(X_{1}, v_{1}\right)$ and $K_{2}=\left(X_{2}, v_{2}\right)$. The " $\Rightarrow$ " part of Equation (25) follows the same scheme as Property 3. Then, $v_{2} \leqslant v_{1}$ implies $X_{1} \subseteq X_{2}$, and it follows that $K_{1} \unlhd_{s} K_{2}$ (and a fortiori, $K_{1} \unlhd_{w} K_{2}$ ). The " $\Leftarrow$ " part of Equation (25) derives from the very definition of $\unlhd_{s}$. $\square$

Property 5 Let $K=(X, v), K^{\prime}=(Y, w) \in \ddot{\Theta}$. Let us suppose that $K \unlhd_{w} K^{\prime}$. Then, we have either (1) $X \subset Y$ or (2) $X=Y$ and $w \leqslant v$. In case (2), we directly have $K \unlhd_{s} K^{\prime}$. Now, let us consider that case (1) holds. Since $K \in \ddot{\Theta}$, there exists $x \in X$ such that $I(x)=v$. Since $X \subset Y$, we have $x \in Y$. But $Y \subseteq \lambda_{w}(I)$ and then $w \leqslant v$. It follows that $K \unlhd_{s} K^{\prime}$. We then have $K \unlhd_{w} K^{\prime} \Rightarrow K \unlhd_{s} K^{\prime}$, and from
Property 1, it comes that $K \unlhd_{w} K^{\prime} \Leftrightarrow K \unlhd_{s} K^{\prime}$. The result follows by transitive reduction of $\unlhd_{w}$ and $\unlhd_{s}$. $\square$

Property 6 Let $K=(X, v), K^{\prime}=(Y, w) \in \dot{\Theta}$. Let us suppose that $K \unlhd_{w} K^{\prime}$. Then, we have either (1) $X \subset Y$ or (2) $X=Y$ and $w \leqslant v$. In case (2), we directly have $K \unlhd_{s} K^{\prime}$. Now, let us consider that case (1) holds. Let $u=\bigvee \leqslant\{v, w\} \in V$. Let us consider $K^{\prime \prime}=(Z, u)$ such that $K^{\prime \prime} \unlhd_{w} K$ and $K^{\prime \prime} \unlhd_{w} K^{\prime}$. Such a node necessarily exists. Let $x \in X$. Then, we have $x \in \lambda_{v}(I)$, and thus $v \leqslant I(x)$. Since $x \in X \subset Y$, we have $x \in \lambda_{w}(I)$, and thus $w \leqslant I(x)$. But then, we have $u=\bigvee \leqslant\{v, w\} \leqslant I(x)$. It follows that $X \subseteq Z$, and since $K^{\prime \prime} \unlhd_{w} K$, we also have $Z \subseteq X$, and then $X=Z$. It comes from the definition of $\dot{\Theta}$ (Equation (17)) that $K^{\prime \prime}=K$, and thus $u=v$. It follows that $w \leqslant \bigvee \leqslant\{v, w\}=u=v$. Then we have $K \unlhd_{s} K^{\prime}$. Consequently, we have $K \unlhd_{w} K^{\prime} \Rightarrow K^{\prime} \unlhd_{s} K$, and from Property 1 , it comes that $K \unlhd_{w} K^{\prime} \Leftrightarrow K^{\prime} \unlhd_{w} K$. The result follows by transitive reduction of $\unlhd_{w}$ and $\unlhd_{s}$. $\square$

Property 7 Let $v \in V$. Let $X \in \mathcal{C}\left[\lambda_{v}(I)\right]$. Let $x \in X$. We have $I(x) \leqslant v$, and then $[x]_{\leftrightarrow_{V}} \subseteq X$. It follows that $X=\bigcup_{x \in X}[x]_{\leftrightarrow_{V}}$. The definition and bijectivity of the two inverse functions $\phi$ and $\phi^{-1}$ defined in Equations (32-33) directly follows from this equality for each value $v \in V$. $\square$

Property 8 Let $\rho(x) \in P$. By definition, we have $x \in \rho(x)$, and then $\rho(x) \neq \emptyset$. Let $y \in \Omega$. We can build sequences of adjacent points of $\Omega$, namely $x=x_{0} \frown \ldots \frown x_{i} \frown \ldots \frown$ $x_{t}=y(t \geq 0)$ such that for any $i \in \llbracket 0, t-1 \rrbracket$, we have $I\left(x_{i}\right)>I\left(x_{i+1}\right)$. Since $\Omega$ is finite, such sequences are also finite. By choosing a sequence of maximal length, we have $x=x_{0} \in \Lambda$, and thus $y \in \rho(x)$. It follows that $\Omega=\bigcup P$. Then, $P$ is a cover of $\Omega$.

Property 9 Let us assume that $v \in \nu((x, y))$. Then, there exists $x^{\prime} \frown y^{\prime}$ such that $x^{\prime} \in \sigma(x)$ and $y^{\prime} \in \sigma(y)$, and $v \in$ $I\left(x^{\prime}\right)^{\downarrow} \cap I\left(y^{\prime}\right)^{\downarrow}$ (from Equations (39-40)). Since $x^{\prime}$ (resp. $y^{\prime}$ ) is in the influence zone of $x$ (resp. $y$ ), then there exists a sequence $x=x_{0} \frown \ldots \frown x_{s}=x^{\prime}$ (resp. $y=y_{0} \frown \ldots \frown x_{u}=$ $y^{\prime}$ ) of points within $\sigma(x)$ (resp. $\sigma(y)$ ) such that for all $i \in$ $\llbracket 0, s \rrbracket$ (resp. $i \in \llbracket 0, u \rrbracket$ ), we have $I\left(x_{i}\right) \geqslant v\left(\right.$ resp. $I\left(y_{i}\right) \geqslant v$ ). By concatenating the first sequence and the reverse second sequence, we build a sequence $x=x_{0} \frown \ldots \frown x_{t}=y(t \geq 1)$ such that for all $i \in \llbracket 0, t \rrbracket, x_{i} \in \sigma(x) \cup \sigma(y)$ and $I\left(x_{i}\right) \geqslant v$. Now, let us assume that there exists a sequence $x=x_{0} \frown$ $\ldots \frown x_{t}=y(t \geq 1)$ such that for all $i \in \llbracket 0, t \rrbracket, x_{i} \in \sigma(x) \cup$ $\sigma(y)$ and $I\left(x_{i}\right) \geqslant v$. Let $j \in \llbracket 0, t \rrbracket$ be the maximal index such that $x_{j} \in \sigma(x)$. Then, we must have $j<t$ and $x_{j+1} \in \sigma(y)$. In particular, we have $\left(x_{j}, x_{j+1}\right) \in E(x, y)$. In addition, we have $v \leqslant I\left(x_{j}\right)$ and $v \leqslant I\left(x_{j+1}\right)$, i.e. $v \in \nu((x, y))$, from Equation (39).

Property 10 Let $K_{1}=\left(X_{1}, v_{1}\right), K_{2}=\left(X_{2}, v_{2}\right) \in \Theta$. Let us suppose that $K_{1} \unlhd K_{2}$. Let $x \in \Lambda$ be the leaf-point such that $\ell_{K_{1}}=\ell(x)$. In particular, we have $x \in X_{1}$. For any other leaf-point $y \in X_{1}$, we have $\ell(x) \leqslant \ell(y)$. Let $z \in \Lambda$ be the leafpoint such that $\ell_{K_{2}}=\ell(z)$. For any other leaf-point $y \in X_{2}$, we have $\ell(z) \leq \ell(y)$. In particular, we have $\ell(z) \leq \ell(x)$, i.e. $\ell_{K_{2}} \leq \ell_{K_{1}}$.

Property 11 Let $K_{1}=\left(X_{1}, v_{1}\right), K_{2}=\left(X_{2}, v_{2}\right) \in \Theta$. Let us suppose that $K_{1} \triangleleft_{s} K_{2}$. We have $K_{1} \unlhd_{s} K_{2}$, and it follows that $v_{2} \leqslant v_{1}$. We also have $X_{1} \subseteq X_{2}$. Let $v_{3} \in V$ with $v_{3} \neq v_{1}$, and let us assume that $v_{2} \leqslant v_{3} \leqslant v_{1}$. Let $K_{3}=\left(X_{3}, v_{3}\right) \in \Theta$ be the node such that $X_{1} \subseteq X_{3}$; such 
node exists and is unique. Then, we must have $X_{3} \subseteq X_{2}$. It comes $K_{1} \unlhd_{s} K_{3} \unlhd_{s} K_{2}$, and $K_{1} \neq K_{3}$. Then, $K_{1} \unlhd_{s} K_{2}$ implies that $K_{3}=K_{2}$, and then $v_{3}=v_{2}$. It follows that $v_{2} \prec v_{1}$.

Property 12 Let $v, w \in V$. Let us assume that $v \leqslant w$. Let $x \in \Lambda^{w}$. Then, from Equation (54) and since $v \leqslant w$, we have $x \in \Lambda^{w}$. Let $(x, y) \in \frown_{\Lambda}^{w}$. We have $w \leqslant I(x)$ and $w \leqslant I(y)$, and then $v \leqslant w \leqslant I(x)$ and $v \leqslant w \leqslant I(y)$. Thus, from Equation (55), we have $(x, y) \in \frown_{\Lambda}^{v}$. It follows that any connected component $Y$ of $\mathfrak{Z}^{w}$ is a subset of a connected component $X$ of $\mathfrak{Z}^{v}$.

\section{Computation of the connected components}

For the three maximal values $v=l, m$ and $n$ of $V$, the 3 matrices $B_{v}$ are defined only by terms $b_{i, j}^{v}=\left(i=j \wedge I\left(x_{i}\right)=v\right)$ (Equation (59)). In other words, they only carry information corresponding to leaves. We have

$C_{n}=B_{n}^{1}=\left(\begin{array}{lllllll}0 & 0 & 0 & 0 & 0 & 0 & 0 \\ 0 & 0 & 0 & 0 & 0 & 0 & 0 \\ 0 & 0 & 0 & 0 & 0 & 0 & 0 \\ 0 & 0 & 0 & 0 & 0 & 0 & 0 \\ 0 & 0 & 0 & 0 & 1 & 0 & 0 \\ 0 & 0 & 0 & 0 & 0 & 0 & 0 \\ 0 & 0 & 0 & 0 & 0 & 0 & 0\end{array}\right)$

$C_{m}=B_{m}^{1}=\left(\begin{array}{lllllll}0 & 0 & 0 & 0 & 0 & 0 & 0 \\ 0 & 1 & 0 & 0 & 0 & 0 & 0 \\ 0 & 0 & 0 & 0 & 0 & 0 & 0 \\ 0 & 0 & 0 & 0 & 0 & 0 & 0 \\ 0 & 0 & 0 & 0 & 0 & 0 & 0 \\ 0 & 0 & 0 & 0 & 0 & 0 & 0 \\ 0 & 0 & 0 & 0 & 0 & 0 & 0\end{array}\right)$

$C_{l}=B_{l}^{1}=\left(\begin{array}{lllllll}1 & 0 & 0 & 0 & 0 & 0 & 0 \\ 0 & 0 & 0 & 0 & 0 & 0 & 0 \\ 0 & 0 & 0 & 0 & 0 & 0 & 0 \\ 0 & 0 & 0 & 0 & 0 & 0 & 0 \\ 0 & 0 & 0 & 0 & 0 & 0 & 0 \\ 0 & 0 & 0 & 0 & 0 & 0 & 0 \\ 0 & 0 & 0 & 0 & 0 & 0 & 0\end{array}\right)$

We then have one valued connected component for each of the three values, namely $(5, n),(2, m)$ and $(1, l)$. In other words, we have $\theta(n)=\{5\}, \theta(m)=\{2\}$ and $\theta(l)=\{1\}$.

For $v=k$, the matrix $B_{k}$ is defined as the disjunction of the three matrices $C_{l}, C_{m}$ and $C_{n}$. This leads to $b_{1,1}^{k}=$ $b_{2,2}^{k}=b_{5,5}^{k}=1$. Moreover, we have $k \in \nu \nabla\left(\left(x_{1}, x_{2}\right)\right)$, that implies $b_{1,2}^{j}=b_{2,1}^{j}=1$. In addition, the leaf-point $x_{3}$ satisfies $I\left(x_{3}\right)=k ;$ then, we set $b_{1,1}^{k}=1$. We have

$C_{k}=B_{k}^{1}=\left(\begin{array}{lllllll}1 & 1 & 0 & 0 & 0 & 0 & 0 \\ 1 & 1 & 0 & 0 & 0 & 0 & 0 \\ 0 & 0 & 1 & 0 & 0 & 0 & 0 \\ 0 & 0 & 0 & 0 & 0 & 0 & 0 \\ 0 & 0 & 0 & 0 & 1 & 0 & 0 \\ 0 & 0 & 0 & 0 & 0 & 0 & 0 \\ 0 & 0 & 0 & 0 & 0 & 0 & 0\end{array}\right)$

Then, we have three valued connected components, namely $(1, k),(3, k)$ and $(5, k)$. In other words, we have $\theta(k)=$
$\{1,3,5\}$. From the analysis of $C_{l}, C_{m}$ and $C_{n}$, it also comes $\varepsilon((k, l))=\{(1,1)\}, \varepsilon((k, m))=\{(1,2)\}$ and $\varepsilon((k, n))=$ $\{(5,5)\}$.

For $v=j$, the matrix $B_{j}$ is defined as the disjunction of the two matrices $C_{l}$ and $C_{m}$. Moreover, we have $j \in \nu \nabla\left(\left(x_{1}, x_{2}\right)\right)$, that implies $b_{1,2}^{j}=b_{2,1}^{j}=1$. We have

$C_{j}=B_{j}^{1}=\left(\begin{array}{lllllll}1 & 1 & 0 & 0 & 0 & 0 & 0 \\ 1 & 1 & 0 & 0 & 0 & 0 & 0 \\ 0 & 0 & 0 & 0 & 0 & 0 & 0 \\ 0 & 0 & 0 & 0 & 0 & 0 & 0 \\ 0 & 0 & 0 & 0 & 0 & 0 & 0 \\ 0 & 0 & 0 & 0 & 0 & 0 & 0 \\ 0 & 0 & 0 & 0 & 0 & 0 & 0\end{array}\right)$

Then, we have one valued connected component, namely $(1, j)$. In other words, we have $\theta(j)=\{1\}$. From the analysis of $C_{l}$ and $C_{m}$, it also comes $\varepsilon((j, l))=\{(1,1)\}$ and $\varepsilon((j, m))=\{(1,2)\}$.

For $v=i$, the matrix $B_{i}$ is defined from the matrix $C_{n}$. Moreover, the leaf-point $x_{6}$ satisfies $I\left(x_{6}\right)=i$; then, we set $b_{6,6}^{k}=1$. We have

$C_{i}=B_{i}^{1}=\left(\begin{array}{lllllll}0 & 0 & 0 & 0 & 0 & 0 & 0 \\ 0 & 0 & 0 & 0 & 0 & 0 & 0 \\ 0 & 0 & 0 & 0 & 0 & 0 & 0 \\ 0 & 0 & 0 & 0 & 0 & 0 & 0 \\ 0 & 0 & 0 & 0 & 1 & 0 & 0 \\ 0 & 0 & 0 & 0 & 0 & 1 & 0 \\ 0 & 0 & 0 & 0 & 0 & 0 & 0\end{array}\right)$

Then, we have two valued connected components, namely $(5, i)$ and $(6, i)$. In other words, we have $\theta(i)=\{5,6\}$. From the analysis of $C_{n}$, it also comes $\varepsilon((i, n))=\{(5,5)\}$.

For $v=h$, the matrix $B_{h}$ is defined from the matrix $C_{k}$. We have

$C_{h}=B_{h}^{1}=\left(\begin{array}{lllllll}1 & 1 & 0 & 0 & 0 & 0 & 0 \\ 1 & 1 & 0 & 0 & 0 & 0 & 0 \\ 0 & 0 & 1 & 0 & 0 & 0 & 0 \\ 0 & 0 & 0 & 0 & 0 & 0 & 0 \\ 0 & 0 & 0 & 0 & 1 & 0 & 0 \\ 0 & 0 & 0 & 0 & 0 & 0 & 0 \\ 0 & 0 & 0 & 0 & 0 & 0 & 0\end{array}\right)$

Then, we have 3 valued connected components, namely $(1, h),(3, h)$ and $(5, h)$. In other words, we have $\theta(h)=$ $\{1,3,5\}$. From the analysis of $C_{k}$, it also comes $\varepsilon((h, k))=$ $\{(1,1),(3,3),(5,5)\}$.

For $v=g$, the matrix $B_{g}$ is defined as the disjunction of the two matrices $C_{j}$ and $C_{k}$. Moreover, the leaf-point $x_{4}$ satisfies $I\left(x_{4}\right)=g$; then, we set $b_{4,4}^{g}=1$. We have

$C_{g}=B_{g}^{1}=\left(\begin{array}{lllllll}1 & 1 & 0 & 0 & 0 & 0 & 0 \\ 1 & 1 & 0 & 0 & 0 & 0 & 0 \\ 0 & 0 & 1 & 0 & 0 & 0 & 0 \\ 0 & 0 & 0 & 1 & 0 & 0 & 0 \\ 0 & 0 & 0 & 0 & 1 & 0 & 0 \\ 0 & 0 & 0 & 0 & 0 & 0 & 0 \\ 0 & 0 & 0 & 0 & 0 & 0 & 0\end{array}\right)$

Then, we have four valued connected components, namely $(1, g),(3, g),(4, g)$ and $(5, g)$. In other words, we have $\theta(g)=$ $\{1,3,4,5\}$. From the analysis of $C_{j}$ and $C_{k}$, it also comes $\varepsilon((g, j))=\{(1,1)\}$ and $\varepsilon((g, k))=\{(1,1),(3,3),(5,5)\}$.

For $v=f$, the matrix $B_{f}$ is defined as the disjunction of the two matrices $C_{h}$ and $C_{i}$. Moreover, we have 
$f \in \nu \nabla\left(\left(x_{1}, x_{6}\right)\right)$, that implies $b_{1,6}^{f}=b_{6,1}^{f}=1$. The same holds for $\nu \nabla\left(\left(x_{2}, x_{3}\right)\right)$ and $\nu \nabla\left(\left(x_{3}, x_{5}\right)\right)$. We have

$B_{f}=\left(\begin{array}{lllllll}1 & 1 & 0 & 0 & 0 & 1 & 0 \\ 1 & 1 & 1 & 0 & 0 & 0 & 0 \\ 0 & 1 & 1 & 0 & 1 & 0 & 0 \\ 0 & 0 & 0 & 0 & 0 & 0 & 0 \\ 0 & 0 & 1 & 0 & 1 & 0 & 0 \\ 1 & 0 & 0 & 0 & 0 & 1 & 0 \\ 0 & 0 & 0 & 0 & 0 & 0 & 0\end{array}\right)$

$B_{f}^{2}=\left(\begin{array}{lllllll}1 & 1 & 1 & 0 & 0 & 1 & 0 \\ 1 & 1 & 1 & 0 & 1 & 1 & 0 \\ 1 & 1 & 1 & 0 & 1 & 0 & 0 \\ 0 & 0 & 0 & 0 & 0 & 0 & 0 \\ 0 & 1 & 1 & 0 & 1 & 0 & 0 \\ 1 & 1 & 0 & 0 & 0 & 1 & 0 \\ 0 & 0 & 0 & 0 & 0 & 0 & 0\end{array}\right)$

$C_{f}=B_{f}^{4}=\left(\begin{array}{lllllll}1 & 1 & 1 & 0 & 1 & 1 & 0 \\ 1 & 1 & 1 & 0 & 1 & 1 & 0 \\ 1 & 1 & 1 & 0 & 1 & 1 & 0 \\ 0 & 0 & 0 & 0 & 0 & 0 & 0 \\ 1 & 1 & 1 & 0 & 1 & 1 & 0 \\ 1 & 1 & 1 & 0 & 1 & 1 & 0 \\ 0 & 0 & 0 & 0 & 0 & 0 & 0\end{array}\right)$

Then, we have one valued connected component, namely $(1, f)$. In other words, we have $\theta(f)=\{1\}$. From the analysis of $C_{h}$ and $C_{i}$, it also comes $\varepsilon((f, h))=\{(1,1),(1,3),(1,5)\}$ and $\varepsilon((f, i))=\{(1,5),(1,6)\}$.

For $v=e$, the matrix $B_{e}$ is defined from the matrix $C_{h}$. Moreover, the leaf-point $x_{7}$ satisfies $I\left(x_{7}\right)=e$; then, we set $b_{7,7}^{e}=1$. Moreover, we have $e \in \nu \nabla\left(\left(x_{2}, x_{3}\right)\right)$, that implies $b_{2,3}^{e}=b_{3,2}^{e}=1$. We have

$B_{e}=\left(\begin{array}{lllllll}1 & 1 & 0 & 0 & 0 & 0 & 0 \\ 1 & 1 & 1 & 0 & 0 & 0 & 0 \\ 0 & 1 & 1 & 0 & 0 & 0 & 0 \\ 0 & 0 & 0 & 0 & 0 & 0 & 0 \\ 0 & 0 & 0 & 0 & 1 & 0 & 0 \\ 0 & 0 & 0 & 0 & 0 & 0 & 0 \\ 0 & 0 & 0 & 0 & 0 & 0 & 1\end{array}\right)$

$C_{e}=B_{e}^{2}=\left(\begin{array}{lllllll}1 & 1 & 1 & 0 & 0 & 0 & 0 \\ 1 & 1 & 1 & 0 & 0 & 0 & 0 \\ 1 & 1 & 1 & 0 & 0 & 0 & 0 \\ 0 & 0 & 0 & 0 & 0 & 0 & 0 \\ 0 & 0 & 0 & 0 & 1 & 0 & 0 \\ 0 & 0 & 0 & 0 & 0 & 0 & 0 \\ 0 & 0 & 0 & 0 & 0 & 0 & 1\end{array}\right)$

Then, we have three valued connected components, namely $(1, e),(5, e)$ and $(7, e)$. In other words, we have $\theta(e)=$ $\{1,5,7\}$. From the analysis of $C_{h}$, it also comes $\varepsilon((e, h))=$ $\{(1,1),(1,3),(5,5)\}$.

For $v=d$, the matrix $B_{d}$ is defined as the disjunction of the two matrices $C_{h}$ and $C_{i}$. Moreover, we have $d \in \nu \nabla\left(\left(x_{1}, x_{6}\right)\right)$, that implies $b_{1,6}^{d}=b_{6,1}^{d}=1$, and this is also the case for $\nu \nabla\left(\left(x_{3}, x_{5}\right)\right)$. We have

$B_{d}=\left(\begin{array}{lllllll}1 & 1 & 0 & 0 & 0 & 1 & 0 \\ 1 & 1 & 0 & 0 & 0 & 0 & 0 \\ 0 & 0 & 1 & 0 & 1 & 0 & 0 \\ 0 & 0 & 0 & 0 & 0 & 0 & 0 \\ 0 & 0 & 1 & 0 & 1 & 0 & 0 \\ 1 & 0 & 0 & 0 & 0 & 1 & 0 \\ 0 & 0 & 0 & 0 & 0 & 0 & 0\end{array}\right)$

$$
C_{d}=B_{d}^{2}=\left(\begin{array}{lllllll}
1 & 1 & 0 & 0 & 0 & 1 & 0 \\
1 & 1 & 0 & 0 & 0 & 1 & 0 \\
0 & 0 & 1 & 0 & 1 & 0 & 0 \\
0 & 0 & 0 & 0 & 0 & 0 & 0 \\
0 & 0 & 1 & 0 & 1 & 0 & 0 \\
1 & 1 & 0 & 0 & 0 & 1 & 0 \\
0 & 0 & 0 & 0 & 0 & 0 & 0
\end{array}\right)
$$

Then, we have two valued connected components, namely $(1, d)$ and $(3, d)$. In other words, we have $\theta(d)=\{1,3\}$. From the analysis of $C_{h}$ and $C_{i}$, it also comes $\varepsilon((d, h))=$ $\{(1,1),(3,3),(3,5)\}$ and $\varepsilon((d, i))=\{(1,6),(3,5)\}$.

For $v=c$, the matrix $B_{c}$ is defined as the disjunction of the two matrices $C_{e}$ and $C_{f}$. Moreover, we have $c \in \nu \nabla\left(\left(x_{2}, x_{7}\right)\right), \nu \nabla\left(\left(x_{5}, x_{6}\right)\right)$ and $\nu \nabla\left(\left(x_{5}, x_{7}\right)\right)$. This implies $b_{5,6}^{c}=b_{6,5}^{c}=1$ (which was already the case), but also $b_{2,7}^{c}=b_{7,2}^{c}=1$ and $b_{5,7}^{c}=b_{7,5}^{c}=1$. We have

$B_{c}=\left(\begin{array}{lllllll}1 & 1 & 1 & 0 & 1 & 1 & 0 \\ 1 & 1 & 1 & 0 & 1 & 1 & 1 \\ 1 & 1 & 1 & 0 & 1 & 1 & 0 \\ 0 & 0 & 0 & 0 & 0 & 0 & 0 \\ 1 & 1 & 1 & 0 & 1 & 1 & 1 \\ 1 & 1 & 1 & 0 & 1 & 1 & 0 \\ 0 & 1 & 0 & 0 & 1 & 0 & 1\end{array}\right)$

$C_{c}=B_{c}^{2}=\left(\begin{array}{lllllll}1 & 1 & 1 & 0 & 1 & 1 & 1 \\ 1 & 1 & 1 & 0 & 1 & 1 & 1 \\ 1 & 1 & 1 & 0 & 1 & 1 & 1 \\ 0 & 0 & 0 & 0 & 0 & 0 & 0 \\ 1 & 1 & 1 & 0 & 1 & 1 & 1 \\ 1 & 1 & 1 & 0 & 1 & 1 & 1 \\ 1 & 1 & 1 & 0 & 1 & 1 & 1\end{array}\right)$

Then, we have one valued connected component, namely $(1, c)$. In other words, we have $\theta(c)=\{1\}$. From the analysis of $C_{e}$ and $C_{f}$, it also comes $\varepsilon((c, e))=\{(1,1),(1,5),(1,7)\}$ and $\varepsilon((c, f))=\{(1,1)\}$.

For $v=b$, the matrix $B_{b}$ is defined as the disjunction of the two matrices $C_{e}$ and $C_{g}$. Moreover, we have $b \in \nu \nabla\left(\left(x_{1}, x_{4}\right)\right)$, that implies $b_{1,4}^{j}=b_{4,1}^{j}=1$, and this is also the case for $\nu \nabla\left(\left(x_{3}, x_{4}\right)\right), \nu \nabla\left(\left(x_{3}, x_{5}\right)\right)$ and $\nu \nabla\left(\left(x_{2}, x_{7}\right)\right)$. We have

$B_{b}=\left(\begin{array}{lllllll}1 & 1 & 1 & 1 & 0 & 0 & 0 \\ 1 & 1 & 1 & 0 & 0 & 0 & 1 \\ 1 & 1 & 1 & 1 & 1 & 0 & 0 \\ 1 & 0 & 1 & 1 & 0 & 0 & 0 \\ 0 & 0 & 1 & 0 & 1 & 0 & 0 \\ 0 & 0 & 0 & 0 & 0 & 0 & 0 \\ 0 & 1 & 0 & 0 & 0 & 0 & 1\end{array}\right)$

$B_{b}^{2}=\left(\begin{array}{lllllll}1 & 1 & 1 & 1 & 1 & 0 & 1 \\ 1 & 1 & 1 & 1 & 1 & 0 & 1 \\ 1 & 1 & 1 & 1 & 1 & 0 & 1 \\ 1 & 1 & 1 & 1 & 1 & 0 & 0 \\ 1 & 1 & 1 & 1 & 1 & 0 & 0 \\ 0 & 0 & 0 & 0 & 0 & 0 & 0 \\ 1 & 1 & 1 & 0 & 0 & 0 & 1\end{array}\right)$

$C_{b}=B_{b}^{4}=\left(\begin{array}{lllllll}1 & 1 & 1 & 1 & 1 & 0 & 1 \\ 1 & 1 & 1 & 1 & 1 & 0 & 1 \\ 1 & 1 & 1 & 1 & 1 & 0 & 1 \\ 1 & 1 & 1 & 1 & 1 & 0 & 1 \\ 1 & 1 & 1 & 1 & 1 & 0 & 1 \\ 0 & 0 & 0 & 0 & 0 & 0 & 0 \\ 1 & 1 & 1 & 1 & 1 & 0 & 1\end{array}\right)$

Then, we have one valued connected component, namely $(1, b)$. In other words, we have $\theta(b)=\{1\}$. From the analysis 
of $C_{e}$ and $C_{g}$, it also comes $\varepsilon((b, e))=\{(1,1),(1,5),(1,7)\}$ and $\varepsilon((b, g))=\{(1,1),(1,3),(1,4),(1,5)\}$.

For $v=a$, we necessarily have

$C_{a}=\left(\begin{array}{lllllll}1 & 1 & 1 & 1 & 1 & 1 & 1 \\ 1 & 1 & 1 & 1 & 1 & 1 & 1 \\ 1 & 1 & 1 & 1 & 1 & 1 & 1 \\ 1 & 1 & 1 & 1 & 1 & 1 & 1 \\ 1 & 1 & 1 & 1 & 1 & 1 & 1 \\ 1 & 1 & 1 & 1 & 1 & 1 & 1 \\ 1 & 1 & 1 & 1 & 1 & 1 & 1\end{array}\right)$

Then, we have one valued connected component, namely $(1, a)$. In other words, we have $\theta(a)=\{1\}$. From the analysis of $C_{b}, C_{c}$ and $C_{d}$, it also comes $\varepsilon((a, b))=\{(1,1)\}$, $\varepsilon((a, c))=\{(1,1)\}$ and $\varepsilon((a, d))=\{(1,1),(1,3)\}$.

\section{Pseudo-code and complexity discussion}

\section{D.1 Input}

The algorithm takes as input:

- $\operatorname{a~graph}(\Omega, \frown)$;

- an ordered value set $(V, \leqslant)$ or its Hasse diagram $(V, \prec)$; and

- a valuation of $I: \Omega \rightarrow V$.

The set $\Omega$ contains vertices $x_{i}$ for $i \in \llbracket 1,|\Omega| \rrbracket$. Each vertex $x_{i}$ can be modelled by its index $i$. As a consequence, $\Omega$ can be represented as an integer vector $\mathcal{V}_{\Omega}$ of length $|\Omega|$ such that $\mathcal{V}_{\Omega}[i]=x_{i}$ for any $i \in \llbracket 1,|\Omega| \rrbracket$. The space cost of $\mathcal{V}_{\Omega}$ is $|\Omega|$.

The set $V$ contains values $v_{i}$ for $i \in \llbracket 1,|V| \rrbracket$. Each value $v_{i}$ can be modelled by its index $i$. As a consequence, $V$ can be represented as an integer vector $\mathcal{V}_{V}$ of length $|V|$ such that $\mathcal{V}_{V}[i]=v_{i}$ for any $i \in \llbracket 1,|V| \rrbracket$. The space cost of $\mathcal{V}_{\Omega}$ is $|V|$.

The valuation $I$ is a mapping between $\Omega$ and $V$. It can be modelled as an integer vector $\mathcal{V}_{I}$ of length $|\Omega|$ such that $I\left(\mathcal{V}_{\Omega}[i]\right)=\mathcal{V}_{V}\left[\mathcal{V}_{I}[i]\right]$ for any $i \in \llbracket 1,|\Omega| \rrbracket$. The space cost of $\mathcal{V}_{I}$ is $|\Omega|$.

The adjacency relation $\frown$ is a part of $\Omega \times \Omega$. It may be modelled as a $|\Omega| \times|\Omega|$ Boolean matrix, but this would require a space cost $|\Omega|^{2}$. In general, each vertex $x_{i}$ has its number of adjacent vertices bounded by a low constant value $m_{\Omega} \ll|\Omega|$, and the induced Boolean matrix is then sparse. Under this hypothesis, it is relevant to handle $\frown$ as a mapping from $\Omega$ to $2^{\Omega}$ that associates to each vertex $x_{i}$ the set of its adjacent vertices. Practically, $\frown$ is then modelled as a vector of integer vectors $\mathcal{V}$ of length $|\Omega|$ such that for each $i \in$ $\llbracket 1,|\Omega| \rrbracket$, the integer vector $\mathcal{V}_{\frown}[i]$ of size $m_{\Omega, i} \leq m_{\Omega}$, is such that for all $j \in \llbracket 1, m_{\Omega, i} \rrbracket$, the vertices $\mathcal{V}_{\Omega}[i]$ and $\mathcal{V}_{\Omega}\left[\mathcal{V}_{\frown}[i][j]\right]$ are adjacent. Note that $\mathcal{V}_{\frown}[i][j]$ is an element of $\mathcal{V}_{\frown}[i]$ if and

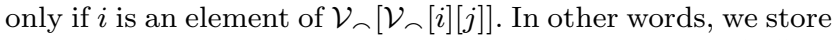
twice each adjacency link. The space cost of $\mathcal{V}$ is $\mathcal{O}(|\Omega|)$.

The order relation $\prec$ is a part of $V \times V$. It may be modelled as a $|V| \times|V|$ Boolean matrix, but this would require a space cost $|V|^{2}$. In general, each value of $V$ has its number of successors bounded by a low constant value $m_{\prec} \ll|V|$, and the induced Boolean matrix is then sparse. Under this hypothesis, it is relevant to handle $\prec$ as a mapping from $V$ to $2^{V}$ that associates to each value $v$ the set of its successors. Practically, $\prec$ is then modelled as a vector of integer vectors $\mathcal{V}_{\prec}$ of length $\mathcal{O}(|V|)$. For each $i \in \llbracket 1,|V| \rrbracket$, the vector $\mathcal{V}_{\prec}[i]$ contains all the indices $j$ such that $\mathcal{V}_{V}[i] \prec \mathcal{V}_{V}\left[\mathcal{V}_{\prec}[i][j]\right]$. The space cost of $\mathcal{V}_{\prec}$ is $\mathcal{O}(|V|)$.

If the provided input is the ordered value set $(V, \leqslant)$ instead $(V, \prec)$, then we have to explicitly compute $(V, \prec)$ from
$(V, \leqslant)$. The time cost for this process depends on the nature of $(V, \leqslant)$. It may vary from $\mathcal{O}(|V|)$ in the simplest cases (e.g. numerical lattices) up to $\mathcal{O}\left(|V|^{\alpha}\right)$ with $2 \leq \alpha \leq 3$ when we have to carry out a transitive reduction on $(V, \leqslant)$ in order to obtain $(V, \prec)$, which has the same time cost as a transitive closure [22].

\section{D.2 Remarks on the partially ordered value sets}

We assume that we are able to compare two values $v, w \in V$, with respect to $\leqslant$, in constant time $\mathcal{O}(1)$. In general, this hypothesis is relevant since most order relations are derived from computable relations (e.g. order relations on Boolean, integer or floating values in standard programming languages). However, in few cases, it may happen that we need the explicit computation and storage of the order relation as a data structure, which generally implies a $\mathcal{O}\left(|V|^{2}\right)$ space cost and possibly a polynomial time cost $\mathcal{O}\left(|V|^{\alpha}\right)$ (with, generally, $2 \leq \alpha \leq 3)$ for its computation if the only input was the Hasse diagram $(V, \prec)$ of the ordered set $(V, \leqslant)$.

The overall space and time cost of the construction of a component-graph is also conditioned by the nature of $(V, \leqslant)$. Indeed, the time cost of the algorithm depends, in part, on the ability to rapidly determine $\nabla \leqslant v^{\downarrow} \cap w^{\downarrow}$ for given two values $v, w \in V$. This is, in particular, the algorithmic foundation of function $\nu \nabla$ (Equation (42)).

In the case where $v$ and $w$ are comparable, i.e. $v \leqslant w$ or $w \leqslant v$, the definition of $\nabla \leqslant v^{\downarrow} \cap w^{\downarrow}$ is simply $\{v\}$ or $\{w\}$, and the issue is then related to the time cost for assessing which of $v$ or $w$ is greater (see paragraph above).

In the case where $v$ and $w$ are non-comparable, i.e. $v \not z w$ and $w \not v$, the access to $\nabla \leqslant v^{\downarrow} \cap w^{\downarrow}$ may be more tricky. In the simplest cases (e.g. for numerical lattices), $\nabla \leqslant v^{\downarrow} \cap w^{\downarrow}$ remains a singleton set, and it can be determined in constant time $\mathcal{O}(1)$ if the Hasse diagram $(V, \prec)$ is available (which is mandatory for building the component-graph). In less favourable cases, the cost for having access to this information for a couple of values $(v, w)$ may be up to $\left|v^{\downarrow} \cup w^{\downarrow}\right|$, that is $\mathcal{O}(|V|)$ in the worst cases. In the sequel, and in particular in Section D.6, we will note this cost $C$. We will keep in mind that $\mathcal{O}(1) \leq C \leq \mathcal{O}(|V|)$, but we encourage the interested readers to consider the construction of componentgraphs in the cases of ordered sets $(V, \leqslant)$ such that $C=\mathcal{O}(1)$.

\section{D.3 Flat zone image computation (see Section 6)}

Building a flat zone image consists of partitioning $\Omega$ into a new set of regions $\Phi$, which are the vertices for a more compact graph. Each of these new vertices $p_{i} \in \Phi$, that can be indexed by an integer value $i \in \llbracket 1,|\Phi| \rrbracket$, gathers one or many vertices of $\Omega$. It is relevant to handle the mapping from $\Phi$ to $2^{\Omega}$ (or equivalently, the inverse non-injective mapping from $\Omega$ to $\Phi$ ) that associates to each vertex $p_{i} \in \Phi$ the set of the corresponding vertices in $\Omega$. Practically, this mapping is modelled as a vector of integers $\mathcal{V}_{\Phi}$ of length $|\Omega|$ such that for each $i \in \llbracket 1,|\Omega| \rrbracket$, the vertex $\mathcal{V}_{\Omega}[i] \in \Omega$ is one of the vertices forming the flat zone corresponding to the vertex $p_{\mathcal{V}_{\Phi}[i]} \in \Phi$ of label $\mathcal{V}_{\Phi}[i]$. The space cost of $\mathcal{V}_{\Phi}$ is $|\Omega|$. The time cost for its construction (Algorithm 1) is $\mathcal{O}(|\Omega|)$.

A new valuation $I_{\Phi}$ on $\Phi$ is induced by the valuation $I$ in $\Omega$ (Equation (31)). It is modelled as an integer vector $\mathcal{V}_{I_{\Phi}}$ of length $|\Phi|$ such that for any $i \in \llbracket 1,|\Omega| \rrbracket$ we have $\mathcal{V}_{I_{\Phi}}\left[\mathcal{V}_{\Phi}[i]\right]=$ 
$\mathcal{V}_{I}[i]$. The space cost of $\mathcal{V}_{I_{\Phi}}$ is $|\Phi|$, and it can be built in parallel to $\mathcal{V}_{I_{\Phi}}$ with no extra-cost (Algorithm 1).

We then have to define the adjacency relation $\frown_{\Phi}$ between the vertices of $\Phi$, induced by the adjacency $\frown$ between those of $\Omega$ (Equation (30)). Practically, $\frown_{\Phi}$ is modelled as a vector of integer vectors $\mathcal{V}_{\frown_{\Phi}}$ of length $|\Phi|$, similarly to $\mathcal{V} \frown$. For each $i \in \llbracket 1,|\Phi| \rrbracket$, the integer vector $\mathcal{V}_{\frown_{\Phi}}[i]$ of size $m_{\Phi, i} \leq m_{\Phi}\left(m_{\Phi}\right.$ may differ from $m_{\Omega}$, but can still be assumed to be a low constant value $\left.m_{\Phi} \ll|\Phi|\right)$, is such that for all $j \in \llbracket 1, m_{\Phi, i} \rrbracket$, the vertices of index $i$ and $\mathcal{V}_{{ }}[i][j]$ are adjacent. Note that $\mathcal{V}_{\frown_{\Phi}}[i][j]$ is an element of $\mathcal{V}_{\frown_{\Phi}}[i]$ if and only if $i$ is an element of $\mathcal{V}_{\frown_{\Phi}}\left[\mathcal{V}{ }_{{ }_{\Phi}}[i][j]\right]$. In other words, we store twice each adjacency link. The space cost of $\mathcal{V}_{\frown \Phi}$ is $\mathcal{O}(|\Phi|)$. The time cost for its construction (Algorithm 2) is $\mathcal{O}(|\Omega|)$.
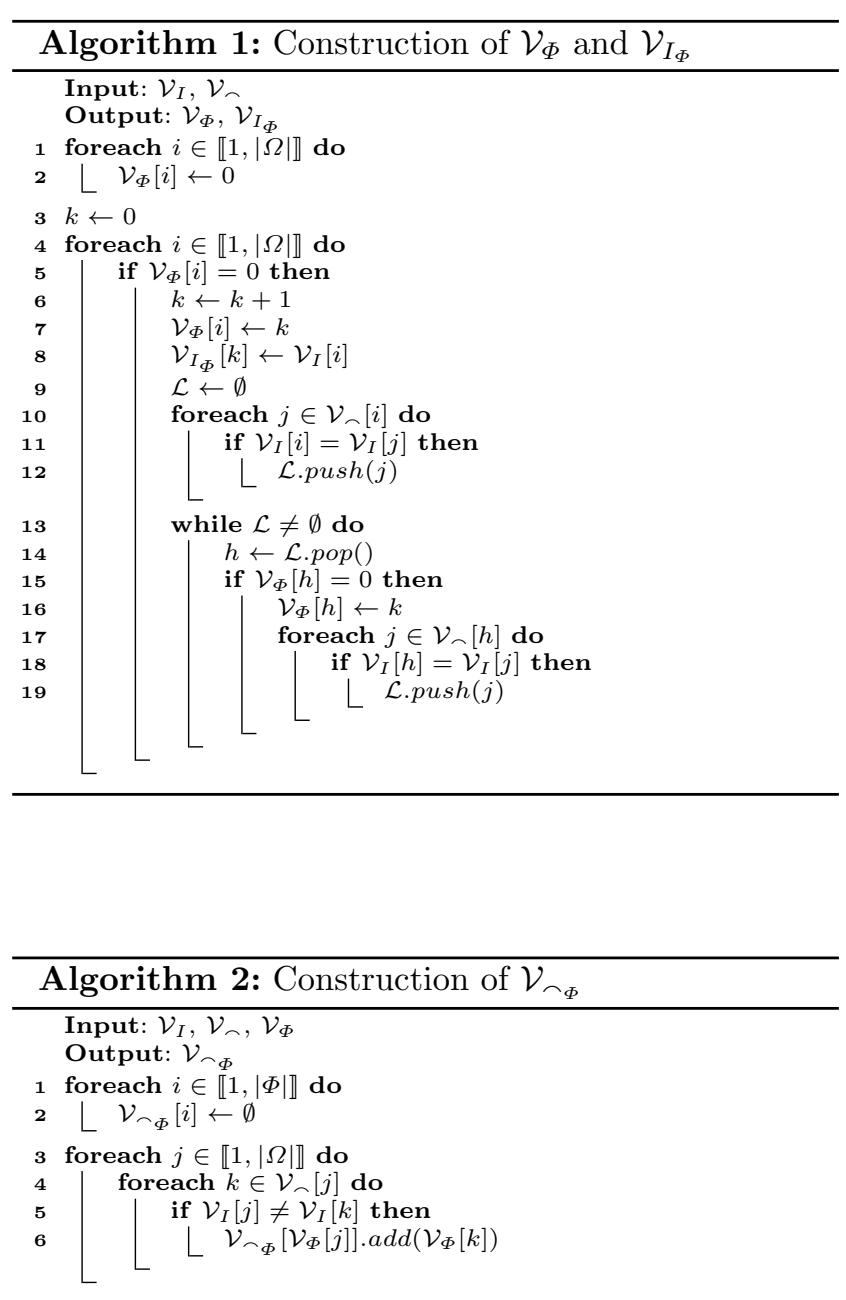

\section{D.4 Leaves computation (Section 7)}

The leaf-points constitute a subset of the vertices of $\Phi$. Each vertex $\lambda_{\ell}$ of this subset $\Lambda \subseteq \Phi$ can be modelled by its index $\ell \in \llbracket 1,|\Lambda| \rrbracket$ (Equation (44)). In particular, each index $\ell$ in $\llbracket 1,|\Lambda| \rrbracket$ is simply a renaming of an index of $\llbracket 1,|\Phi| \rrbracket$ with respect to $\Phi$. We model this injective mapping from $\llbracket 1,|\Lambda| \rrbracket$ to $\llbracket 1,|\Phi| \rrbracket$ by defining a vector of integers $\mathcal{V}_{\Lambda}$ of length $|\Lambda|$ such that for any $\ell \in \llbracket 1,|\Lambda| \rrbracket$, the leaf-point $\lambda_{\ell}$ in $\Lambda$ is equal to the

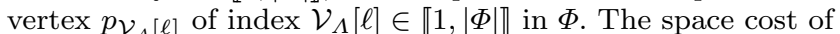
$\mathcal{V}_{\Lambda}$ is $|\Lambda|$. The time cost for its construction (Algorithm 3) is $\mathcal{O}(|\Phi|)$.

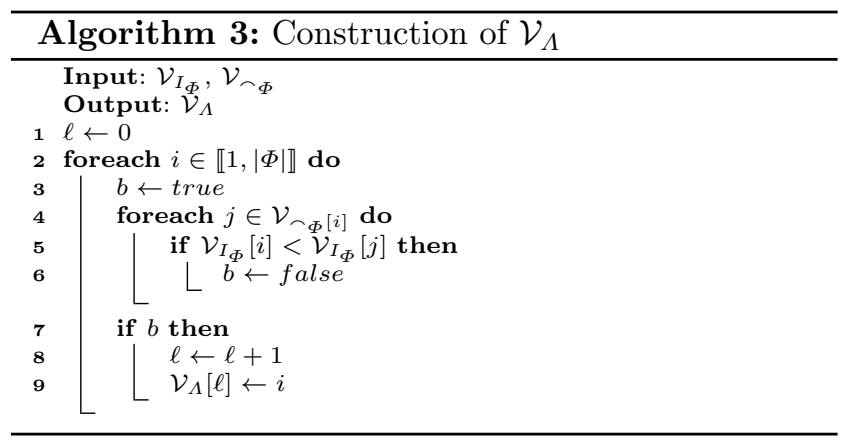

\section{D.5 Influence zones computation (Section 8)}

Building the influence zones of the flat zone image consists of assigning to each vertex of $\Phi$ the label of the leaf-point of $\Lambda$ that defines the influence zone where it lies. We model this mapping from $\llbracket 1,|\Phi| \rrbracket$ to $\llbracket 1,|\Lambda| \rrbracket$ by defining a vector of integers $\mathcal{V}_{\rho}$ of length $|\Phi|$ such that the vertex $p_{i}$ in $\Phi$ of index $i \in \llbracket 1,|\Phi| \rrbracket$ lies in the influence zone $\rho\left(p_{\left.\mathcal{V}_{\Lambda}\left[\mathcal{V}_{\rho}[i]\right]\right)}\right)$ of the leafpoint $\lambda_{\mathcal{V}_{\rho}[i]}$ of $\Lambda$ of index $\mathcal{V}_{\rho}[i]$ in $\llbracket 1,|\Lambda| \rrbracket$. The space cost of $\mathcal{V}_{\rho}$ is $|\Phi|$. The time cost for its construction (Algorithm 4) is $\mathcal{O}(|\Phi|)$.

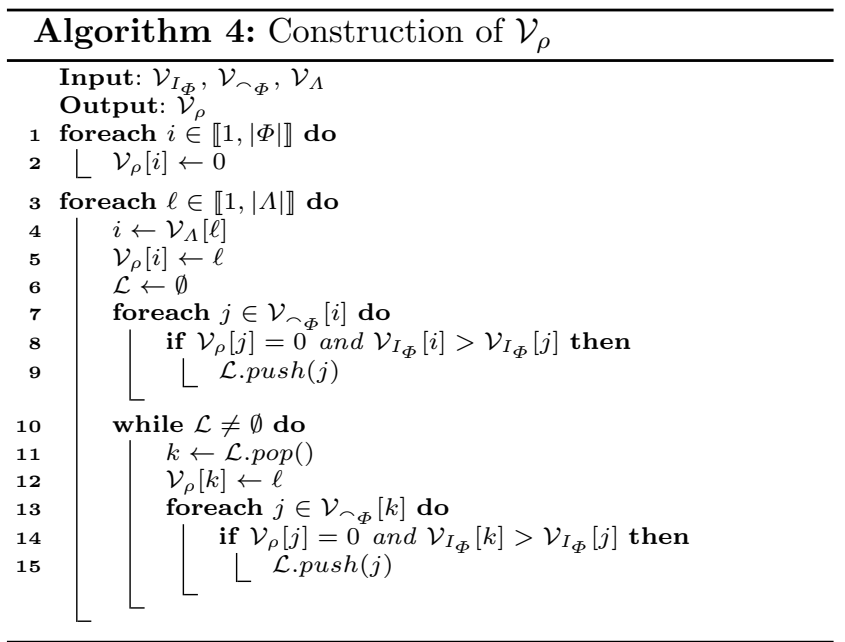

\section{D.6 Influence zones graph construction (Section 9)}

The vertices of the influence zone graph are the influence zones of the leaf-points or, equivalently, the leaf-points themselves. The only task for building this graph is then to define the adjacency relation $\frown \Lambda$ between the vertices of $\Lambda$, induced 
by the adjacency $\frown_{\Phi}$ between those of $\Phi$ (Equation (38)). Practically, $\Omega_{\Lambda}$ is modelled as a vector of integer vectors $\mathcal{V}_{\frown_{\Lambda}}$ of length $|\Lambda|$, similarly to $\mathcal{V}_{\frown_{\Phi}}$. For each $\ell \in \llbracket 1,|\Lambda| \rrbracket$, the integer vector $\mathcal{V}_{-\Lambda}[\ell]$ of size $m_{\Lambda, \ell} \leq m_{\Lambda}$ ( $m_{\Lambda}$ may differ from $m_{\Phi}$, but can still be assumed to be a low constant value $\left.m_{\Lambda} \ll|\Lambda|\right)$, is such that for all $k \in \llbracket 1, m_{\Lambda, \ell} \rrbracket$, the vertices of index $\ell$ and $\mathcal{V}_{\frown_{A}}[\ell][k]$ are adjacent. Note that $\mathcal{V}_{\frown_{A}}[\ell][k]$ is an element of $\mathcal{V}_{-}[\ell]$ if and only if $\ell$ is an element of $\mathcal{V}_{\frown_{\Lambda}}\left[\mathcal{V}_{\frown_{\Lambda}}[\ell][k]\right]$. In other words, we store twice each adjacency link. The space cost of $\mathcal{V}_{\Lambda_{\Lambda}}$ is $\mathcal{O}(|\Lambda|)$. The time cost for its construction (Algorithm 5) is $\mathcal{O}(|\Phi|)$.

It is then necessary to define the valuation $\nu$ of the edges of the graph $(\Lambda, \frown \Lambda)$ (Equation (39)). As discussed in Section 9 , it is not necessary to store the whole function $\nu$. In particular, a less costly function, namely $\nu \nabla$ can be considered (Equation (42)). We observe that $\nu \nabla$ will then be involved in the construction of the connected components of the thresholded graph of $(\Lambda, \frown \Lambda)$ at each value $v \in V$ (Equation (59)). In this context, it is indeed relevant to associate to each value $v \in V$ the set of all edges $e$ of $\frown \Lambda$ such that $v \in \nu \nabla(e)$, instead of associating to each edge $e$ the set of values $\nu \nabla(e)$. In other words, we model the mapping $\nu \nabla^{-1}$ instead of $\nu \nabla$. This is done by defining a vector of integer vectors $\mathcal{V}_{\nu}$ of length $|V|$. For each $i \in \llbracket 1,|V| \rrbracket$, the integer vector $\mathcal{V}_{\nu}[i]$ provides all the couples of indices $(\ell, k)$ such that $\left(\mathcal{V}_{\Lambda}[\ell], \mathcal{V}_{\Lambda}[k]\right)$ is an edge of $\frown_{\Lambda}$ that satisfies $\mathcal{V}_{V}[i] \in \nu \nabla\left(\left(\mathcal{V}_{\Lambda}[\ell], \mathcal{V}_{\Lambda}[k]\right)\right)$. The space cost of $\mathcal{V}_{\nu}$ is $\mathcal{O}(|V|+|\Phi|)$, since the vector $\mathcal{V}_{\nu}$ is of length $|V|$, whereas the total amount of edges stored is at most the same as for $\frown_{\Phi}$, multiplied by a low value (assumed constant) that bounds $\left|\nabla \leqslant u^{\downarrow} \cap v^{\downarrow}\right|$ for any $u, v \in V$. The time cost for its construction (Algorithm 6) is $(|V|+|\Phi| . C)$ (see Section D.2 for the definition of $C$ ). Note that for each $i \in \llbracket 1,|V| \rrbracket$, the size of $\mathcal{V} \nu[i]$ is in average $\mathcal{O}(|\Phi| /|V|)$, that can be considered as a constant value $\beta$. In particular, ensuring that $\mathcal{V} \nu[i]$ has no extra occurrence of each element (that is equivalent to maintaining the set sorted) has a time $\operatorname{cost} \mathcal{O}(\beta \log \beta)$ whereas ensuring that each of its elements is a maximal one has a time cost $\mathcal{O}\left(\beta^{2}\right)$. These costs are then assumed to remain constant, and not to impact the overall cost $(|V|+|\Phi| . C)$.

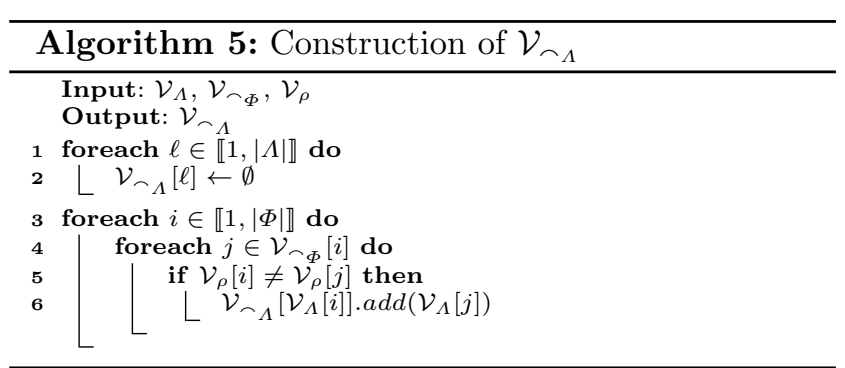

\section{D.7 Connected component computation (Section 11.1)}

At this stage, we have to build the connected components of the thresholded influence zone graphs for each value $v$ of $V$. As stated in Section 11.1, this consists of computing, for each value $v$, the matrix $C_{v}$, which is a Boolean matrix of size $|\Lambda| \times$ $|\Lambda|$ corresponding to the reflexive-transitive closure of the adjacency matrix $A_{v}$ of the thresholded influence zone graph at value $v$. More efficiently, this adjacency matrix $A_{v}$ can be replaced by a matrix $B_{v}$ (Equation (59)) defined from the

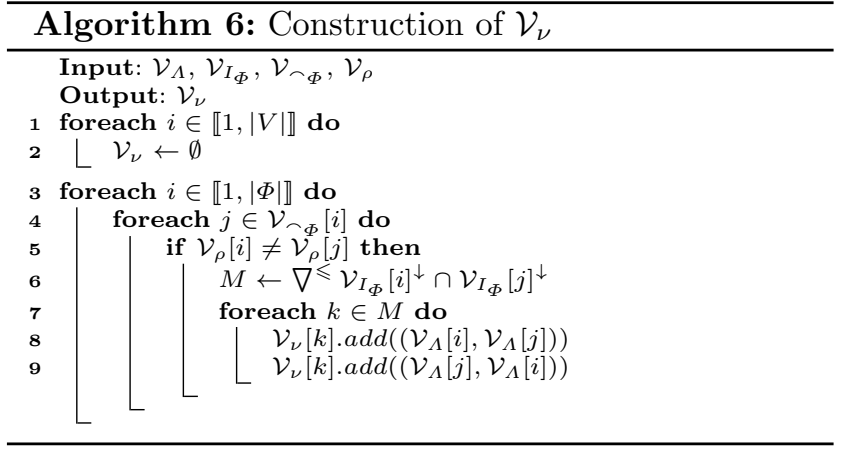

matrices $C_{w}$ for any values $w \succ v$, the set of edges belonging to $\nu \nabla^{-1}(\{v\})$ (Section D.6), and the set of leaves of value $v$. In particular, the $B_{v}$ and $C_{v}$ matrices are built in a recursive, top-down fashion from the maximal values of $(V, \leqslant)$ to its minimium $\perp$.

For each value $v$, the matrix $C_{v}$ is most often a sparse matrix. Indeed, it is a $|\Lambda| \times|\Lambda|$ Boolean matrix, but the number of rows / columns containing at least one non-zero value is the number $N_{v} \leq|\Lambda|$ of leaf-points of value $u \geqslant v$. Then, $C_{v}$ can be handled and stored as a $N_{v} \times N_{v}$ matrix. In addition, each remaining row / column of $C_{v}$ can contain a number of non-zero values that is lower than $N_{v}$. Storing the indices of these elements is indeed sufficient for preserving the information of the whole matrix. Then, $C_{v}$ is stored as a vector of integer vectors $\mathcal{V}_{C_{v}}$ of length $N_{v}$. For each $i \in \llbracket 1, N_{v} \rrbracket, C_{v}[i]$ is a vector that contains as first element $C_{v}[i][0]$ the index $i$ of a leaf-point $\mathcal{V}_{\Lambda}[i]$ of $\Lambda$ of value $u \geqslant v$. Note that the $C_{v}[i][0]$ indices are sorted from the lowest (for $i=1$ ) to the greatest (for $i=N_{v}$ ). For a given vector $C_{v}[i]$, the elements $C_{v}[i][j]$ for $j>0$ are the indices of the leaf-points $\mathcal{V}_{\Lambda}\left[\mathcal{V}_{C_{v}}[i][j]\right]$ which are connected to $\mathcal{V}_{\Lambda}[i]$ in the thresholded influence zone graph at value $v$. Note that the $C_{v}[i][j]$ indices are sorted from the lowest (for $j=1$ ) to the greatest. The space cost of $\mathcal{V}_{C_{v}}$ is equal to the number of non-zero values of $C_{v}$ and is in particular in $\mathcal{O}\left(N_{v}^{2}\right)$. The time cost for its computation is $\mathcal{O}\left(N_{v}^{\alpha}\right)$ with $2 \leq \alpha \leq 3$, and $\alpha$ close to 2 in many cases. Indeed, it proceeds in two steps.

First, we have to build the vector of integer vectors $\mathcal{V}_{B_{v}}$ of the matrix $B_{v}$ (which is structured the same way as $\mathcal{V}_{C_{v}}$ ). It is simply the element-wise disjunction of the matrices $C_{w}$ for $w \succ v$, and two other matrices corresponding to the vector of $\mathcal{V}_{\nu}[a]$ with $v=\mathcal{V}_{V}[a]$ and the subset of indices $\ell$ of $\mathcal{V}_{\Lambda}$ such that $\mathcal{V}_{I_{\Phi}}\left[\mathcal{V}_{I_{A}}[\ell]\right]=v$, respectively. These last two matrices are structured the same way as $\mathcal{V}_{C_{v}}$. By assuming that the number of successors for the relation $\prec$ is bounded by a low constant value $m^{\prec}$, the cost for building this disjunction is $\mathcal{O}\left(N_{v}^{2}\right)$, since it corresponds to the merging of several sorted lists while maintaining the values ordered.

Second, we have to compute the reflexive-transitive closure of $\mathcal{V}_{B_{v}}$. This is a simple matrix product procedure that consists of computing $B_{v}^{2^{k}}$ for $k>0$ until convergence. In the worst case, the number of iterations is $\log _{2} N_{v}$. In average, it is in general a low constant value. Each iteration consists of a self-product which time cost is, in theory, $N_{v}^{3}$. However, since $B_{v}$ is initially reflexive, the non-zero values in $B_{v}$ remain nonzero in the next product matrices. Concerning the other, zero elements, only those corresponding to a couple of row and column that was modified at the previous step need to be recomputed (otherwise, the value remains necessarily zero). Finally, only the zero elements corresponding to rows and / 
or columns that were modified at the previous steps need an update, which cost is in the worst case linear with respect to the number of non-zero values in the corresponding row and column. In practice, this computation can be stopped when a couple of equal values is identified in the couple of row / column. The overall cost for this computation is then $\mathcal{O}\left(N_{v}^{\alpha}\right)$ with $2 \leq \alpha \leq 3$, and $\alpha$ close to 2 in many cases.

\section{D.8 Hasse diagram enrichement (Sections 11.2 and $11.3)$}

The final step for building the component-graph consists of defining the function $\theta$ (resp. $\varepsilon$ ) that provides, for each value $v$ of $V$ (resp. each edge $(v, w)$ of $\prec$ ) the set of the labels of the canonical leaf-points corresponding to the valued connected components at value $v$ (resp. the couples of labels of canonical leaf-points that are linked by the 4 relation between values $v$ and $w$ in the component-graph).

On the one hand, the mapping $\theta$ is modelled by a vector of integer vectors $\mathcal{V}_{\theta}$ of length $|V|$. For each $i \in \llbracket 1,|V| \rrbracket$, the vector $\mathcal{V}_{\theta}[i]$ contains all the indices $j$ such that $\mathcal{V}_{\Lambda}[j] \in$ $\theta\left(\mathcal{V}_{V}[i]\right)$. The space cost of $\mathcal{V}_{\theta}$ is $|\Theta|$. The time cost for the computation of each of its $|V|$ vectors $\mathcal{V}_{\theta}[i]$ (from $\mathcal{V}_{C_{v}}$ ) is $N_{v}$ with $v=\mathcal{V}_{V}[i]$ since it is sufficient to scan the vector $\mathcal{V}_{C_{v}}$ and to add to $\mathcal{V}_{\theta}[i]$ all the indices $\mathcal{V}_{C_{v}}[j][0]$, for $1 \leq j \leq N_{v}$, such that $\mathcal{V}_{C_{v}}[j][0]=\mathcal{V}_{C_{v}}[j][1]$.

On the other hand, the mapping $\varepsilon$ is modelled by a vector of vectors of vectors of couples of integers $\mathcal{V}_{\varepsilon}$ of length $|V|$. For each valid couple of indices $(i, j)$, the vector $\mathcal{V}_{\varepsilon}[i][j]$ contains all the couples $(\ell, k)$ of indices such that $\mathcal{V}_{\Phi}\left[\mathcal{V}_{\Lambda}[\ell]\right]$ and $\mathcal{V}_{\Phi}\left[\mathcal{V}_{\Lambda}[k]\right]$ are leaf-points satisfying $\mathcal{V}_{I_{\Phi}}\left[\mathcal{V}_{\Phi}\left[\mathcal{V}_{\Lambda}[\ell]\right]\right]=\mathcal{V}_{V}[i]$ and $\mathcal{V}_{I_{\Phi}}\left[\mathcal{V}_{\Phi}\left[\mathcal{V}_{\Lambda}[k]\right]\right]=\mathcal{V}_{V}[j]$ and $\left(k, \mathcal{V}_{V}[j]\right) \triangleleft\left(\ell, \mathcal{V}_{V}[i]\right)$. The space cost of $\mathcal{V}_{\theta}$ is $|\varangle|$. The time cost for the computation of each of its $|\prec|$ vectors $\mathcal{V}_{\varepsilon}[i][j]$ (from $\mathcal{V}_{\theta}[j]$ and $\mathcal{V}_{C_{\mathcal{V}_{V}[i]}}$ ) is $N_{v}$ with $v=\mathcal{V}_{V}[i]$. Indeed, for each $a$ in $\mathcal{V}_{\theta}[j]$ one has to add the couple $\left(\mathcal{V}_{C_{\mathcal{V}_{V}}}[b][1], a\right)$ such that $\mathcal{V}_{C_{\mathcal{V}_{V}}}[b][0]=a$.

\section{References}

1. Najman, L., Talbot, H. (eds.): Mathematical Morphology: From Theory to Applications. ISTE/J. Wiley \& Sons (2010)

2. Salembier, P., Oliveras, A., Garrido, L.: Anti-extensive connected operators for image and sequence processing. IEEE Transactions on Image Processing 7, 555-570 (1998)

3. Monasse, P., Guichard, F.: Scale-space from a level lines tree. Journal of Visual Communication and Image Representation 11, 224-236 (2000)

4. Salembier, P., Garrido, L.: Binary partition tree as an efficient representation for image processing, segmentation and information retrieval. IEEE Transactions on Image Processing 9, 561-576 (2000)

5. Passat, N., Naegel, B.: Component-hypertrees for image segmentation. In: ISMM, International Symposium on Mathematical Morphology, Proceedings, Lecture Notes in Computer Science, vol. 6671, pp. 284-295. Springer (2011)

6. Perret, B., Cousty, J., Tankyevych, O., Talbot, H., Passat, N.: Directed connected operators: Asymmetric hierarchies for image filtering and segmentation. IEEE Transactions on Pattern Analysis and Machine Intelligence 37(6), 1162-1176 (2015)
7. Naegel, B., Passat, N.: Component-trees and multi-value images: A comparative study. In: International Symposium on Mathematical Morphology (ISMM), Lecture Notes in Computer Science, vol. 5720, pp. 261-271. Springer (2009)

8. Passat, N., Naegel, B.: An extension of component-trees to partial orders. In: International Conference on Image Processing (ICIP), pp. 3981-3984 (2009)

9. Passat, N., Naegel, B.: Component-trees and multivalued images: Structural properties. Journal of Mathematical Imaging and Vision 49, 37-50 (2014)

10. Kurtz, C., Naegel, B., Passat, N.: Connected filtering based on multivalued component-trees. IEEE Transactions on Image Processing 23, 5152-5164 (2014)

11. Naegel, B., Passat, N.: Toward connected filtering based on component-graphs. In: International Symposium on Mathematical Morphology (ISMM), Lecture Notes in Computer Science, vol. 7883, pp. 350-361. Springer (2013)

12. Naegel, B., Passat, N.: Colour image filtering with component-graphs. In: International Conference on Pattern Recognition (ICPR), pp. 1621-1626 (2014)

13. Carlinet, E., Géraud, T.: MToS: A tree of shapes for multivariate images. IEEE Transactions on Image Processing 24, 5330-5342 (2015)

14. Xu, Y., Géraud, T., Najman, L.: Connected filtering on tree-based shape-spaces. IEEE Transactions on Pattern Analysis and Machine Intelligence 38, 1126-1140 (2016)

15. Grossiord, É., Naegel, B., Talbot, H., Passat, N., Najman, L.: Shape-based analysis on component-graphs for multivalued image processing. In: International Symposium on Mathematical Morphology (ISMM), Lecture Notes in Computer Science, vol. 9082, pp. 446-457. Springer (2015)

16. Grossiord, É., Naegel, B., Talbot, H., Najman, L., Passat, N.: Shape-based analysis on component-graphs for multivalued image processing. Mathematical Morphology Theory and Applications (In Press)

17. Passat, N., Naegel, B., Kurtz, C.: Implicit componentgraph: A discussion. In: International Symposium on Mathematical Morphology (ISMM), Lecture Notes in Computer Science, vol. 10225, pp. 235-248. Springer (2017)

18. Salembier, P., Serra, J.: Flat zones filtering, connected operators, and filters by reconstruction. IEEE Transactions on Image Processing 4, 1153-1160 (1995)

19. Kong, T. Y., Rosenfeld, A.: Digital topology: Introduction and survey. Computer Vision, Graphics, and Image Processing 48, 357-393 (1989)

20. Adams, R., Bischof, L.: Seeded region growing. IEEE Transactions on Pattern Analysis and Machine Intelligence 16, 641-647 (1994)

21. Jones, R.: Connected filtering and segmentation using component trees. Computer Vision and Image Understanding 75, 215-228 (1999)

22. Aho, A. V., Garey, M. R., Ullman, J. D.: The transitive reduction of a directed graph. SIAM Journal on Computing 1, 131-137 (1972) 